\title{
The Impact of Global Climate Change and Global Warming on Public Health and Welfare Cost From Exposure to Environmental Risks
}

\author{
Rodica Pripoaie \\ rodica.pripoaie@ugal.ro \\ Dunarea de Jos University of Galati, Romania
}

\begin{abstract}
The aim of the paper is to presents the impact of global climate change and global warming on public health and welfare cost from exposure to environmental risks. The paper shows the implications of greenhouse gases on the environment with potentially damaging effects on ecosystems, biodiversity and the means of human subsistence. This study attempts to empirically examine the dynamic causal relationships between greenhouse gases, mortality rate and public health and welfare cost from exposure to environmental risks, using the time-series data for the period 1999-2016.
\end{abstract}

Keywords - greenhouse gases, welfare cost, global warming.

JEL Code: F 64, I 19, O 44.

\section{Introduction}

Air pollution is a consequence of increasing urbanization and industrialization. Greenhouse gas (GHG) emissions contribute to accelerating climate change. The effect of greenhouse gas emissions on the environment is worrying, especially because in recent years they have had a fulminate development despite international agreements that have taken some decisions on their limitation. Greenhouse gases remain in the atmosphere for a few years to thousands of years and they have a worldwide impact. The global climate change has an impact on mortality rate, public health and welfare cost from exposure to environmental risks. Also, the greenhouse gases have a potentially damaging effect on ecosystems, biodiversity and of human subsistence. It is a dynamic causal relationship between greenhouse gases, mortality rate and public health and welfare cost, shows by the time-series data for the period $1999-2016$.

\section{Short literature review}

"Ambient air pollution has been associated with a multitude of health effects, including mortality, respiratory and cardiovascular hospitalizations, changes in lung function, asthma attacks, and days lost from work (Bates 1995a; Pope 1996, 2000; Samet et al. 2000a, 2000b; Segala 1999). These studies have been performed in multiple cities around the United States and internationally using various designs and statistical methods." (Wong, E, Goblke, J, Griffith, W, Farrow, S and Faustman, E, 2004)

Greenhouse gases contribute to global warming because they absorb the sun's energy and prevents the release of harmful gases in space. Thus, while global warming will lead to what will lead to the disappearance of life on Earth.

Eurostat considers that "each greenhouse gas has a different capacity to cause global warming, depending on its radioactive properties, molecular weight and the length of time it remains in the atmosphere.

The global warming potential (GWP) of each gas is defined in relation to a given weight of carbon dioxide for a set time period (for the purpose of the Kyoto Protocol a period of 100 years). 
GWPs are used to convert emissions of greenhouse gases to a relative measure (known as carbon dioxide equivalents: $\mathrm{CO}_{2}$-equivalents). The weighting factors currently used is the following:

- $\quad$ carbon dioxide $=1$,

- methane $=25$,

- $\quad$ nitrous oxide $=298$,

- $\quad$ sulphur hexafluoride $=22800$;

- hydrofluorocarbons and perfluorocarbons comprise a large number of different gases that have different GWPs. "(https://ec.europa.eu/eurostat/statisticsexplained/pdfscache/1180.pdf)

The biggest emitters and the sector that produces the most emissions are:

- energy, fuel combustion and fugitive emissions from fuels, which also includes transport;

- industrial processes;

- agriculture;

- land use, land use change and forestry (LULUCF);

- waste management.

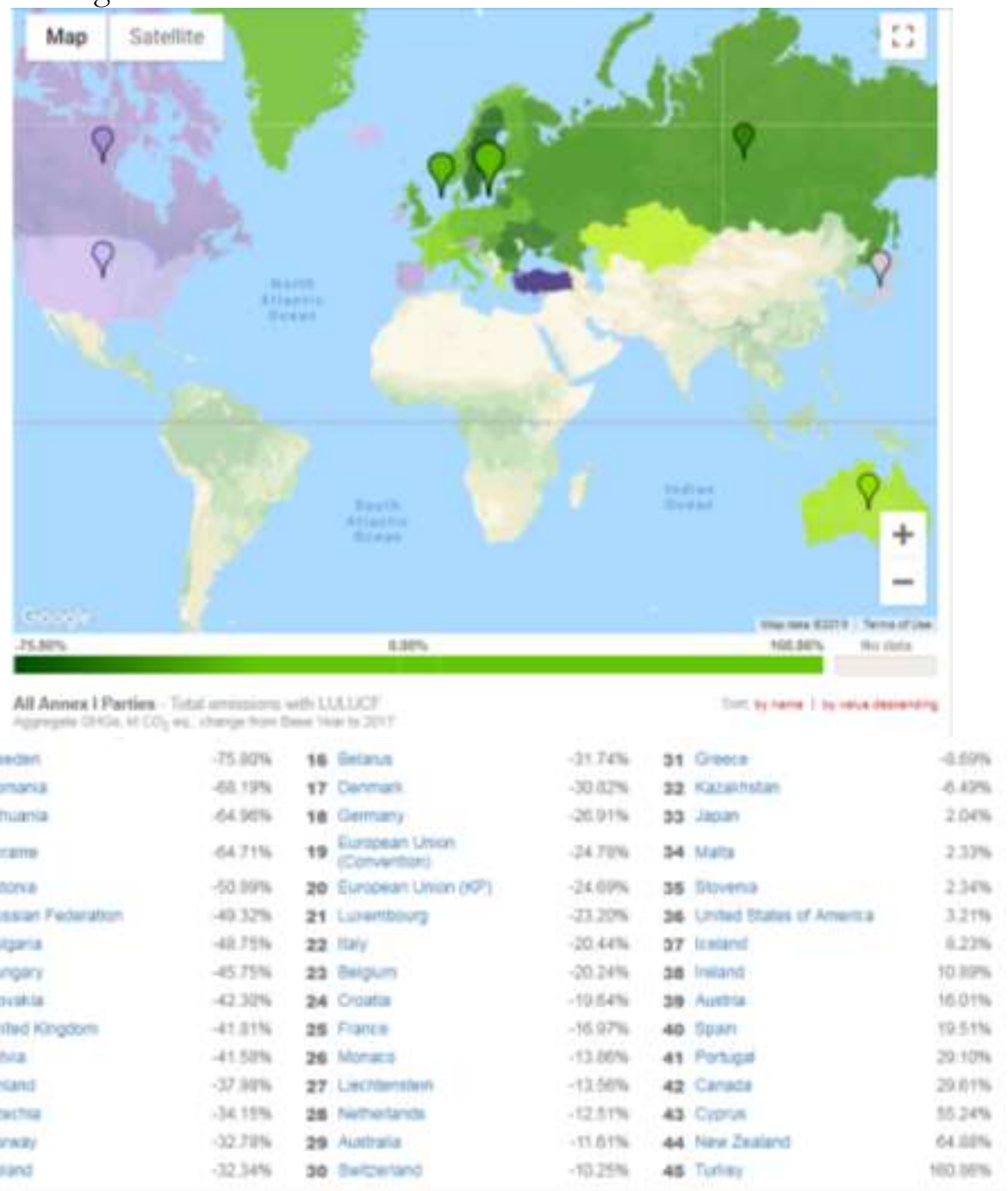

Figure 1. The evolution of total emissions with LULUCF

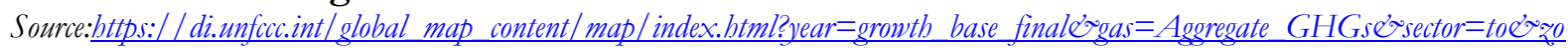
om $=2 \&$ lat $=30 \Leftrightarrow \operatorname{lng}=15$ 
As the figure above shows, $\mathrm{CO}_{2}$ is the greenhouse gas that is emitted the most, produced by human activities. Most of emissions of carbon dioxide come from burning fossil fuels, with additional contributions from deforestation, generically said LULUCF.

Experts say that if greenhouse gas emissions continue at their rate in 2017, the Earth's surface temperature could exceed historic values from 2047 with potentially damaging effects on ecosystems, biodiversity and the means of human subsistence.

\section{Actually problems of global climate change and global warming}

Globally, deforestation of the equatorial and tropical forests is so great that specialists say that every minute there is a forest of the size of 36 football fields, and that deforestation contributes about $6-12 \%$ of total $\mathrm{CO}_{2}$ emissions.

In Romania, massive deforestation in recent years has led to irreparable environmental damage. Deforestation causes floods, soil erosion, landslides, and the disappearance of animals from their natural environment. Thus, the Carpathian bears from what was a few years ago, Wild Carpathian, do not find enough food in the forest because of deforestation and often go out to cities like Brasov, Buşteni or Sinaia to eating. The once destroyed ecosystem is hard to recover or even impossible.

Climate change affecting the worldwide in various forms, depending on the region. Greenhouse gases create the greenhouse effect leading to global warming and the appearance of extreme weather events, such as floods, tornadoes in areas where so far no record of such weather events, forest fires and changing seasons.

Also at this adds overuse everything is made of plastic, such as bags, bottles, glasses, packages are found everywhere in the oceans and forests.

It requires the emergence of new industries and factories that do not pollute, because pollution and smog around big cities affect people's health, it can also cause more and more allergic disorders, respiratory, lung, lung cancer and thus to reduce its duration and quality of life.

Also, outdated and polluting industries and very old cars pollute the environment. The solution is to replace them with electric cars, clean, and larger scale use of electricity produced from renewable sources such as wind turbines and giving the energy produced by power plants that are highly polluting.

Many organizations, such as Greenpeace fight for environmental protection, but their efforts should be enhanced and all countries understand that pollution and global warming effects worldwide and they at some point will be irreversible, and the costs for each country will be much higher, both financially and humanely, by affecting the health of the population and the quality of life.

\section{Measures to reducing the greenhouses gases emissions}

\section{1.}

Since 2008 the European Union set a target to minimize GHG emissions by $20 \%$ by 2020 compared to year 1990. Also, in 2015 there was a decrease of $22 \%$ of GHG emissions compared with 1990 levels. Then, in 2014, the EU set a new objective of decreasing GHG emissions by at least $40 \%$ by 2030 compared to 1990 levels, but in 2017 it estimates there will be a decrease of about $30 \%$ in 2030 .

\section{Carbon offsetting by forests}

The forests have power to absorb $\mathrm{CO}_{2}$ and so they fight with climate change and offset carbon from the land use sector.

The EU forests have 182 million hectares, covering 43\% of its land area. The forest's surface and their weight can vary from one state to another and they absorb the equivalent of $10.9 \%$ of total EU GHG emissions each year.

4.2. 
In 2018 the European Commission adopted a strategic long-term vision for minimize GHG emissions by 2050, named "A Clean Planet for all", which aims to create a vision and plan to develop new and innovative industries and businesses and not targets.

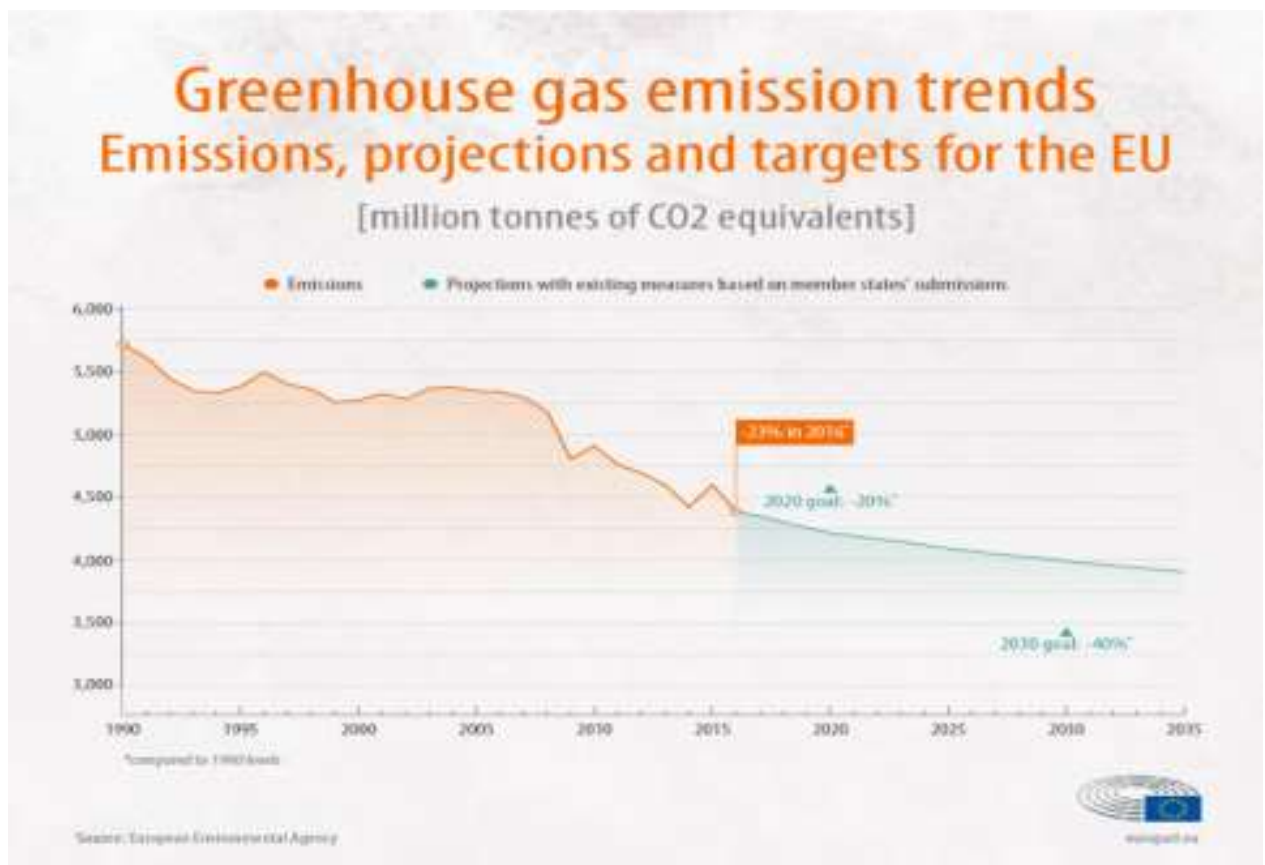

Sources: bttp:/ / www.europarl.europa.eu/ resources/library/images/20180709PHT07512/20180709PHT07512 original.jpg

At the beginning of this year, 2019, the European Parliament was adopted for the first time ever regulation on $\mathrm{CO}_{2}$ emissions for trucks to decreasing the transport emissions. Thus, it follows that to be reduced by $30 \%$, by the year 2030, with an intermediate target of $15 \%$, by the year 2025 .

Also by 2025 , producers of trucks will be ensuring that $2 \%$ market share of the sales with zero-and-low-emission vehicles, as electric or hybrid trucks. This regulation will reduce the pollution and to improve air quality. The future of clean trucks is because this sector produces about $15 \%$ of $\mathrm{EU} \mathrm{CO}_{2}$ emissions and road transport is the only sector where greenhouse gas emissions are still higher than in 1990.

\section{Methodology}

The statistical data have been taken from the Eurostat https://ec.europa.eu/eurostat/, from the United Nations Framework Convention on Climate Change (UNFCCC) https://unfccc.int/ and from OECD https://data.oecd.org/. Data collected about GHG and mortality and welfare costs has been processed with EViews 10, graphically represented and interpreted.

Based on the https://data.oecd.org/ database, the following summary of the GHG emission from 1990-2017 is presented. 


\section{Table 1. Summary of GHG emissions}

\begin{tabular}{|c|c|c|c|}
\hline & \multicolumn{3}{|c|}{ Emissions, in $\mathrm{kt} \mathrm{CO}_{2}$ equivalent } \\
\hline & Base year & 2000 & Last Inventory Year (2017) \\
\hline $\mathrm{CO}_{2}$ emissions without LULUCF & $4,549,519.8$ & $2,567,085.7$ & $2,655,476.7$ \\
\hline $\mathrm{CO}_{2}$ net emissions/removals by LULUCF & $-283,361.2$ & $-752,235.5$ & $-740,044.3$ \\
\hline $\mathrm{CO}_{2}$ net emissions/removals with LULUCF & $4,266,158.6$ & $1,814,850.2$ & $1,915,432.4$ \\
\hline GHG emissions without LULUCF & $5,812,621.3$ & $3,369,786.3$ & $3,489,856.4$ \\
\hline GHG net emissions/removals by LULUCF & $-243,833.2$ & $-707,459.8$ & $-694,162.0$ \\
\hline GHG net emissions/removals with LULUCF & $5,568,788.1$ & $2,662,326.5$ & $2,795,694.4$ \\
\hline \multirow[t]{3}{*}{ Indirect $\mathrm{CO}_{2}$} & $1,889.6$ & $1,185.8$ & 727.6 \\
\hline & \multicolumn{3}{|c|}{ Changes in emissions, in percent } \\
\hline & From Base year to 2000 & $\begin{array}{c}\text { From } 2000 \text { to Last Inventory } \\
\text { Year (2017) }\end{array}$ & $\begin{array}{l}\text { From Base year to Last } \\
\text { Inventory Year (2017) }\end{array}$ \\
\hline $\mathrm{CO}_{2}$ emissions without LULUCF & $-43.57 \%$ & $3.44 \%$ & $-41.63 \%$ \\
\hline $\mathrm{CO}_{2}$ net emissions/removals by LULUCF & $165.47 \%$ & $-1.62 \%$ & $161.17 \%$ \\
\hline $\mathrm{CO}_{2}$ net emissions/removals with LULUCF & $-57.46 \%$ & $5.54 \%$ & $-55.10 \%$ \\
\hline GHG emissions without LULUCF & $-42.03 \%$ & $3.56 \%$ & $-39.96 \%$ \\
\hline GHG net emissions/removals by LULUCF & $190.14 \%$ & $-1.88 \%$ & $184.69 \%$ \\
\hline GHG net emissions/removals with LULUCF & $-52.19 \%$ & $5.01 \%$ & $-49.80 \%$ \\
\hline
\end{tabular}

\begin{tabular}{|c|c|c|c|}
\hline & \multicolumn{3}{|c|}{ Average annual growth rates, in percent per year } \\
\hline & From Base year to 2000 & $\begin{array}{c}\text { From } 2000 \text { to Last Inventory } \\
\text { Year (2017) }\end{array}$ & $\begin{array}{l}\text { From Base year to Last } \\
\text { Inventory Year (2017) }\end{array}$ \\
\hline $\mathrm{CO}_{2}$ emissions without LULUCF & $-5.56 \%$ & $0.20 \%$ & $-1.97 \%$ \\
\hline $\mathrm{CO}_{2}$ net emissions/removals by LULUCF & $10.26 \%$ & $-0.10 \%$ & $3.62 \%$ \\
\hline $\mathrm{CO}_{2}$ net emissions/removals with LULUCF & $-8.19 \%$ & $0.32 \%$ & $-2.92 \%$ \\
\hline GHG emissions without LULUCF & $-5.31 \%$ & $0.21 \%$ & $-1.87 \%$ \\
\hline GHG net emissions/removals by LULUCF & $11.24 \%$ & $-0.11 \%$ & $3.95 \%$ \\
\hline GHG net emissions/removals with LULUCF & $-7.11 \%$ & $0.29 \%$ & $-2.52 \%$ \\
\hline
\end{tabular}

The base year under the Climate Change Convention is 1990 except for Bulgaria (1988), Hungary (average of 1985 to 1987), Poland (1988), Romania (1989) and Slovenia (1986), as defined by decisions 9/CP.2 and 11/CP.4.

Source: bttps:/ / unfccc.int/

The evolution of GHG emissions with or without LULUCF in the period 1990-2017 is presented in the followings graphics:

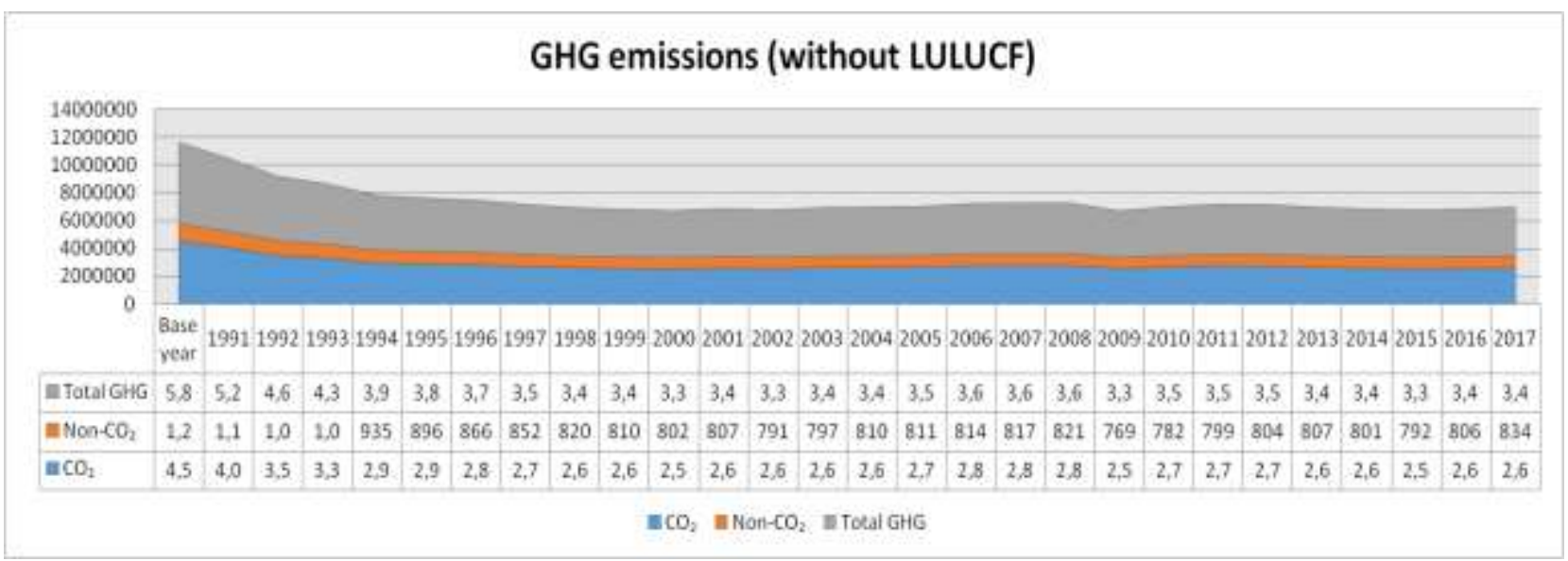

Figure 1. The evolution of the GHG emissions without LULUCF

Source: Author, by using the bttps: / unfccc.int/ data (2019) 


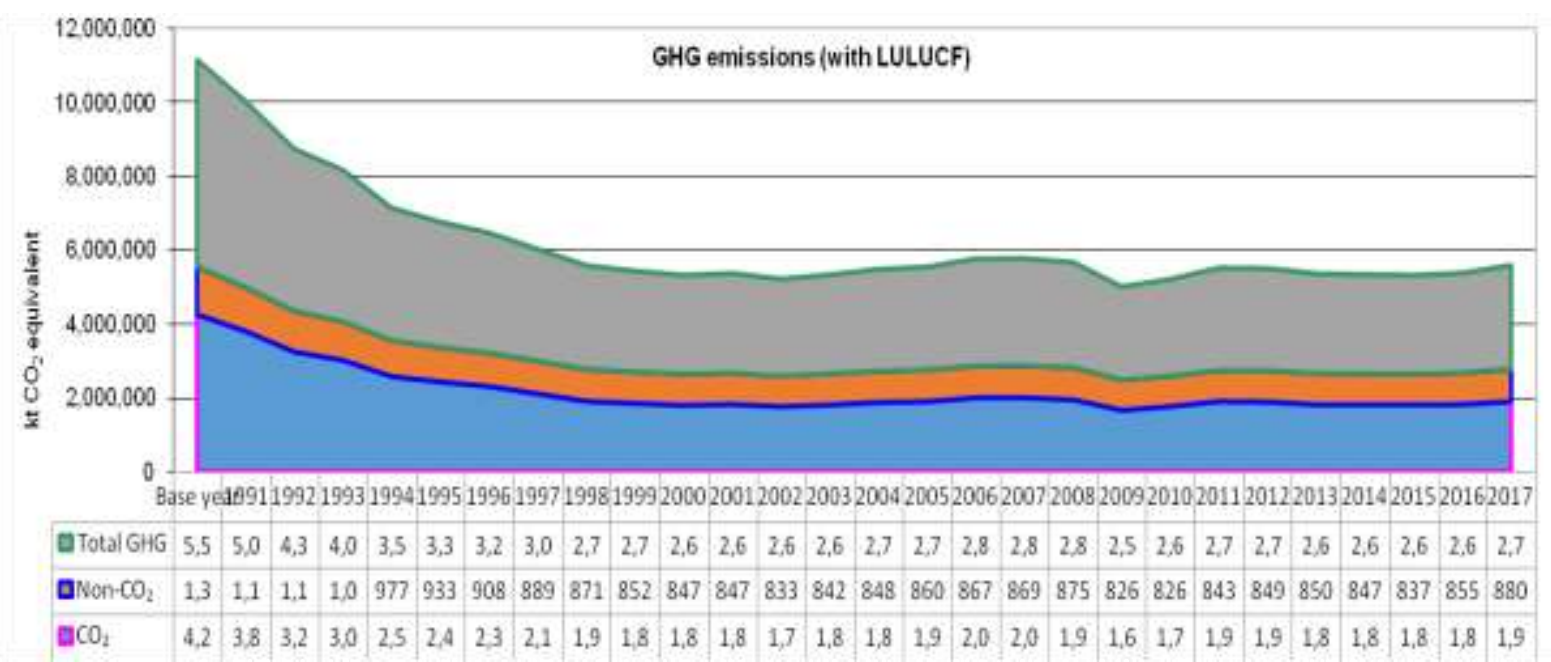

Figure 2. The evolution of the GHG emissions with LULUCF

Source: Author, by using the bttps: / / unfccc.int/ data (2019)

The evolution of GHG emissions / removals changes from 1990 to 2017 can be summarized as follows:

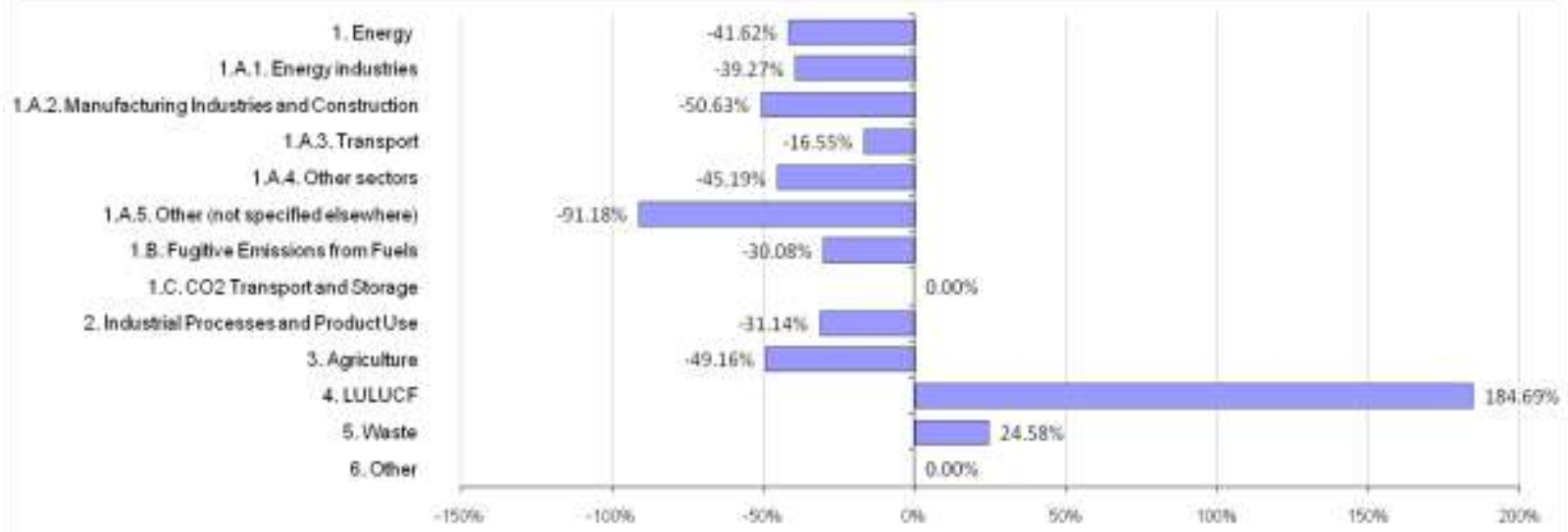

Figure 3. The evolution of the GHG emissions/removals

Source: https://unfccc.int/

Also, the GHG emissions by gas in 2017 compared with base year 1990 are presented in the followings graphics, with or without LULUCF:

1990 (without LULUCF)

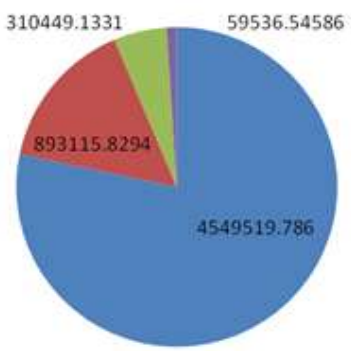

$=\mathrm{CO}_{2}-78.27 \%$

n $\mathrm{CH}_{4}-15.37 \%$

$=\mathrm{N}_{2} \mathrm{O}-5.34 \%$

n Aggregate f-gases - $1.02 \%$
2017 (without LULUCF)

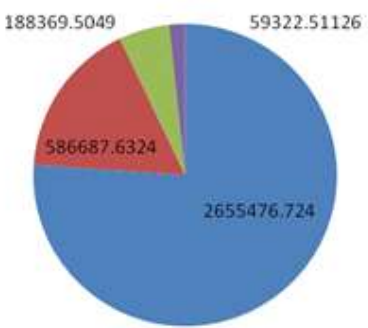

$=\mathrm{CO}_{2}-76.09 \%$

m $\mathrm{CH}_{4}-16.81 \%$

$=\mathrm{N}_{2} \mathrm{O} \cdot 5.40 \%$

$=$ Aggregate F-gases - $1.70 \%$

Figure 4. The evolution of the GHG emissions by gas without LULUCF Source: https://unfccc.int/ 


\section{0 (with LULUCF)}

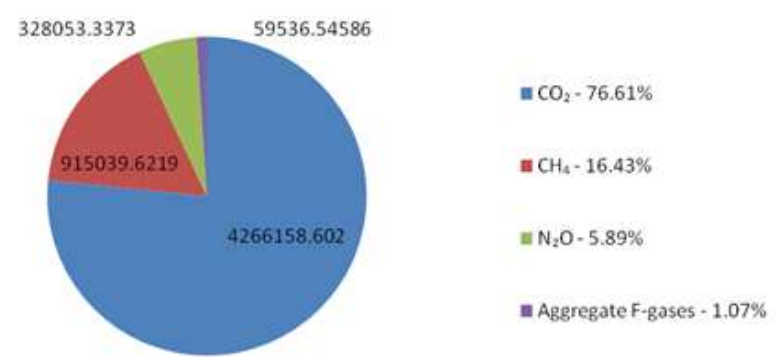

\section{7 (with LULUCF)}

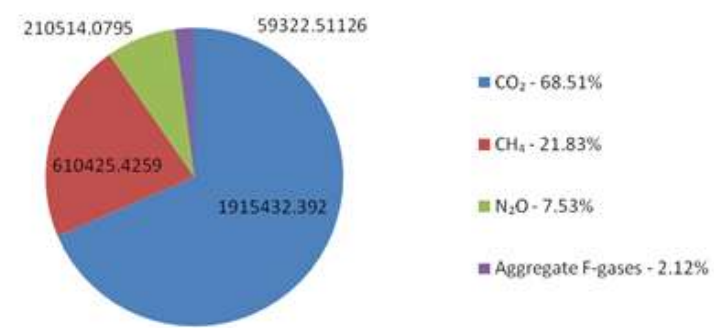

Figure 5. The evolution of the GHG emissions by gas with LULUCF Source: https://unfccc.int/

And the GHG emissions by sector without LULUCF in 2017 compared with base year 1990 are presented in the followings graphics:

1990

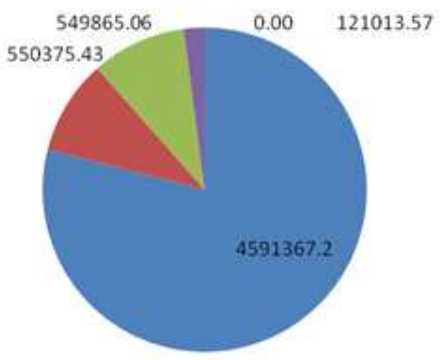

Energy - $78.99 \%$

Industrial Processes and Product Use - $9.47 \%$

$=$ Agriculture $-9.46 \%$

Waste- $2.08 \%$

$=$ Other $-0.00 \%$
2017

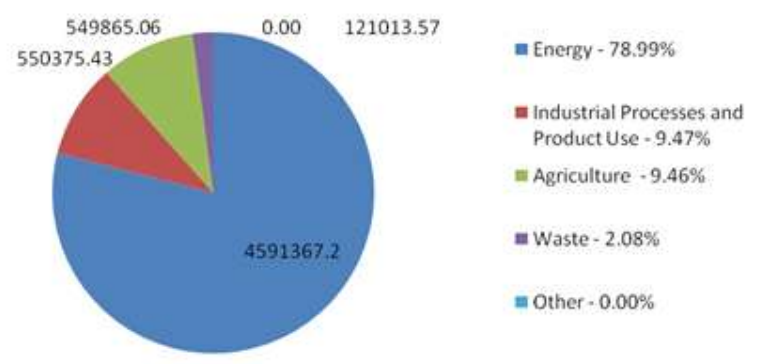

Figure 6. The evolution of the GHG emissions by sector without LULUCF Source: https://unfcccint/

\section{Results and discussions}

The statistical data collected about GHG and mortality and welfare costs has been processed with EViews 10, graphically represented and interpreted.

GHG data series and mortality and welfare costs data series are used to determine descriptive indicators and statistical or graphical estimation of econometric models. Evolution of the two variables analyzed in the period 1999-2016 is presented using EViews 10, as follows: 


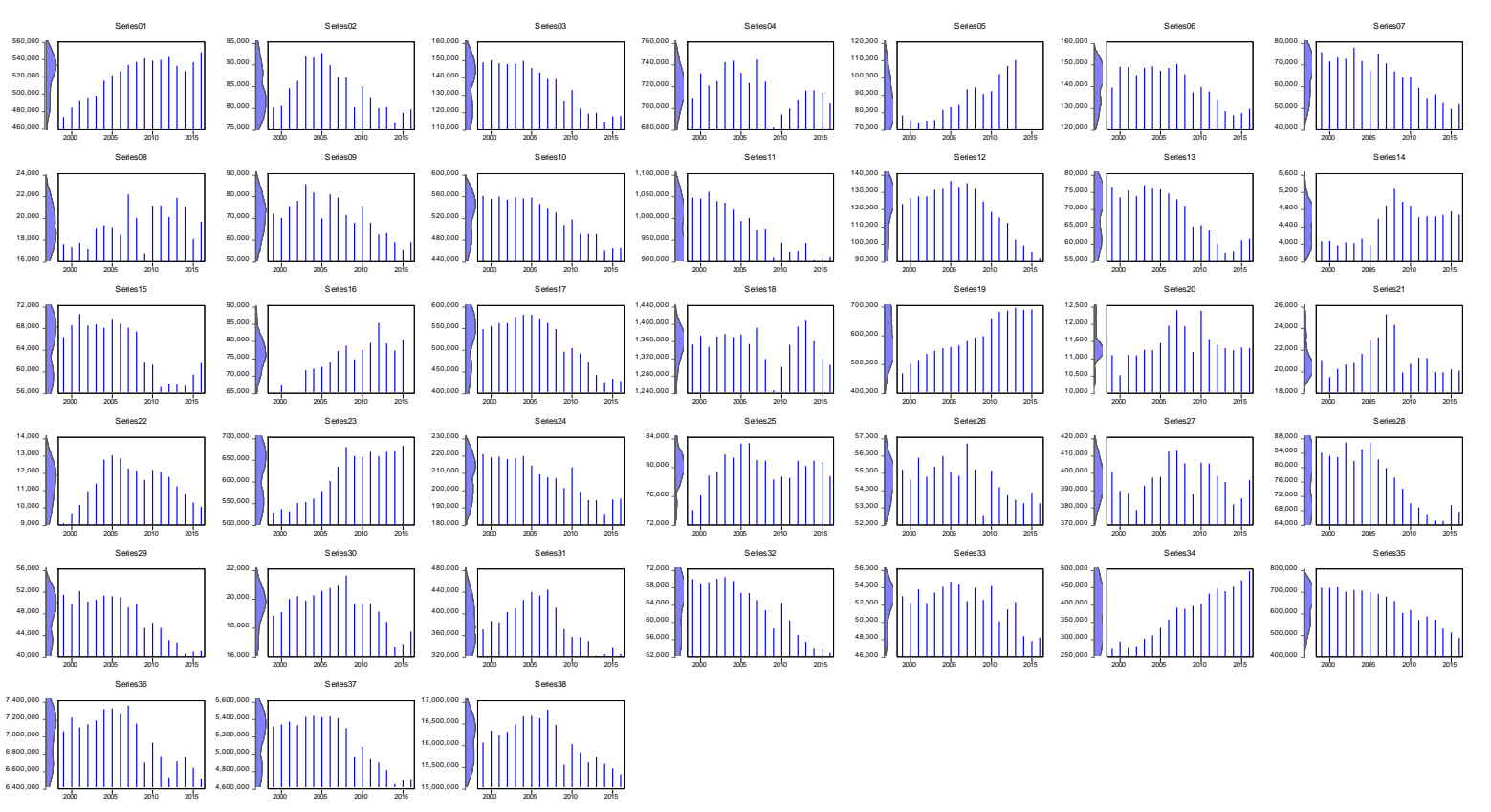

Figure 7. The evolution of the GHG emissions with EViews 10 for OECD statistical data Source: Author, by using the https://stats.oecd.org/ (2019)
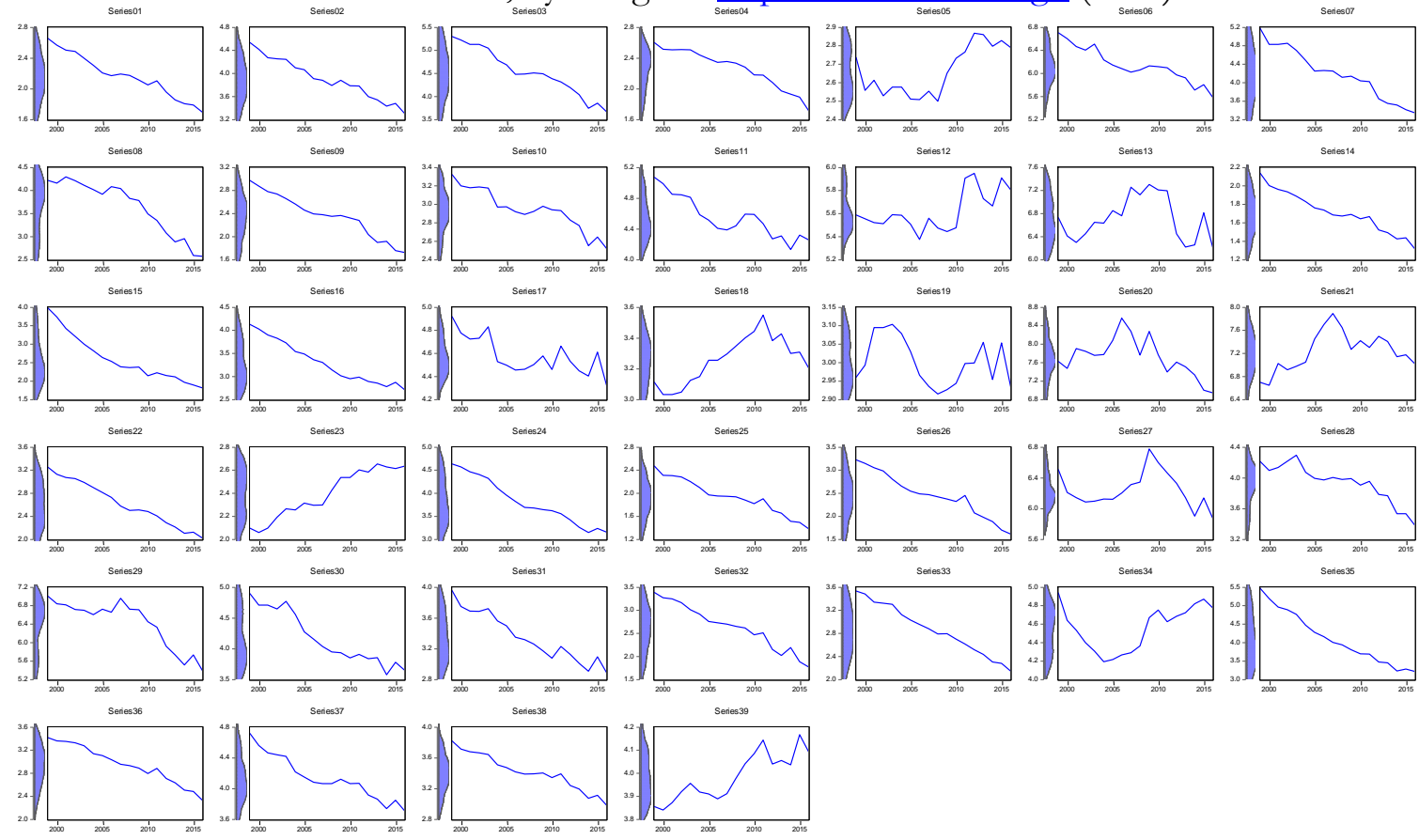

Figure 8. The evolution of the mortality and welfare costs with EViews 10 for OECD statistical data

Source: Author, by using the https://stats.oecd.org/ (2019)

Or if data is analyzed as multiple series, the evolution is following: 

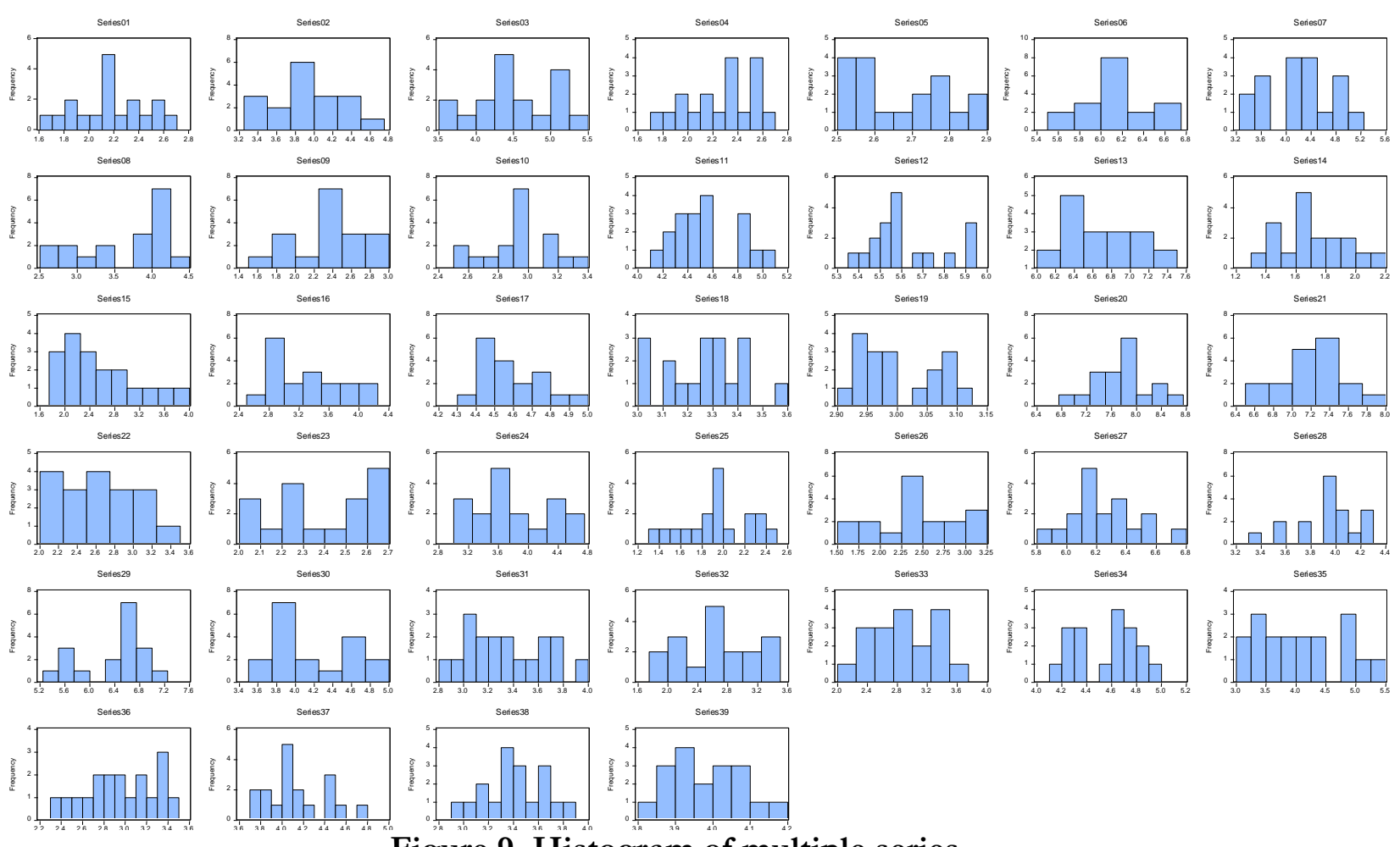

Figure 9. Histogram of multiple series

Source: Author, by using the EViews 10

Descriptive indicators for mortality and welfare costs data series for OECD Europe and OECD Total are those in the following tables:

Table 2. Summary of descriptive indicators for mortality and welfare costs in OECD

Dependent Variable: OECD EUROPE2

\section{Europe}

Method: Least Squares

Date: 04/11/19 Time: 00:15

Sample: 19992016

Included observations: 18

\begin{tabular}{lclll}
\hline \multicolumn{1}{c}{ Variable } & Coefficient & Std. Error & t-Statistic & Prob. \\
\hline \hline \multicolumn{1}{c}{ OECD_EUROPE } & $7.22 \mathrm{E}-07$ & $1.63 \mathrm{E}-07$ & 4.437487 & 0.0004 \\
& 0.429809 & 0.837371 & 0.513284 & 0.6148 \\
\hline \hline R-squared & 0.551711 & Mean dependent var & 4.139944 \\
Adjusted R-squared & 0.523693 & S.D. dependent var & 0.284714 \\
S.E. of regression & 0.196495 & Akaike info criterion & -0.311916 \\
Sum squared resid & 0.617767 & Schwarz criterion & -0.212986 \\
Log likelihood & 4.807244 & Hannan-Quinn criter. & -0.298275 \\
F-statistic & 19.69129 & Durbin-Watson stat & 0.452208 \\
Prob(F-statistic) & 0.000414 & & & \\
\hline \hline
\end{tabular}

Source: Author, by using the EViews 10 
Table 3. Summary of descriptive indicators mortality and welfare costs in OECD Total Dependent Variable: OECD TOTAL2

Method: Least Squares

Date: 04/11/19 Time: 00:46

Sample: 19992016

Included observations: 18

\begin{tabular}{lrlrr}
\hline \hline \multicolumn{1}{c}{ Variable } & Coefficient & Std. Error & t-Statistic & Prob. \\
\hline \hline OECD__TOTAL & $3.05 E-07$ & $9.64 \mathrm{E}-08$ & 3.164128 & 0.0060 \\
\multicolumn{1}{c}{ C } & -1.497109 & 1.552867 & -0.964094 & 0.3493 \\
\hline \hline R-squared & 0.384892 & Mean dependent var & 3.414333 \\
Adjusted R-squared & 0.346448 & S.D. dependent var & 0.233968 \\
S.E. of regression & 0.189145 & Akaike info criterion & -0.388162 \\
Sum squared resid & 0.572416 & Schwarz criterion & -0.289232 \\
Log likelihood & 5.493462 & Hannan-Quinn criter. & -0.374521 \\
F-statistic & 10.01170 & Durbin-Watson stat & 0.471592 \\
Prob(F-statistic) & 0.006014 & & \\
\hline \hline
\end{tabular}

Source: Author, by using the EViews 10

Ordinary covariance analysis between the GHG series and mortality and welfare costs is as follows and we can observe it appears that the two variables are linear correlated.

Figure 10.

OECD__TOTAL2 vs. Variables (Partialled on Regressors)

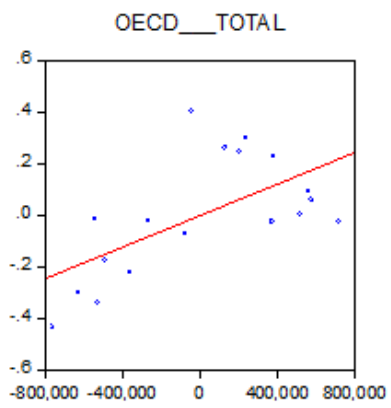

C

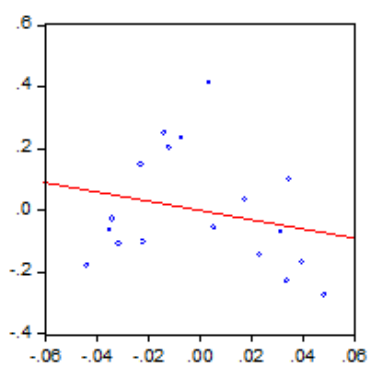

Source: Author, by using the EViews 10
Figure 11.

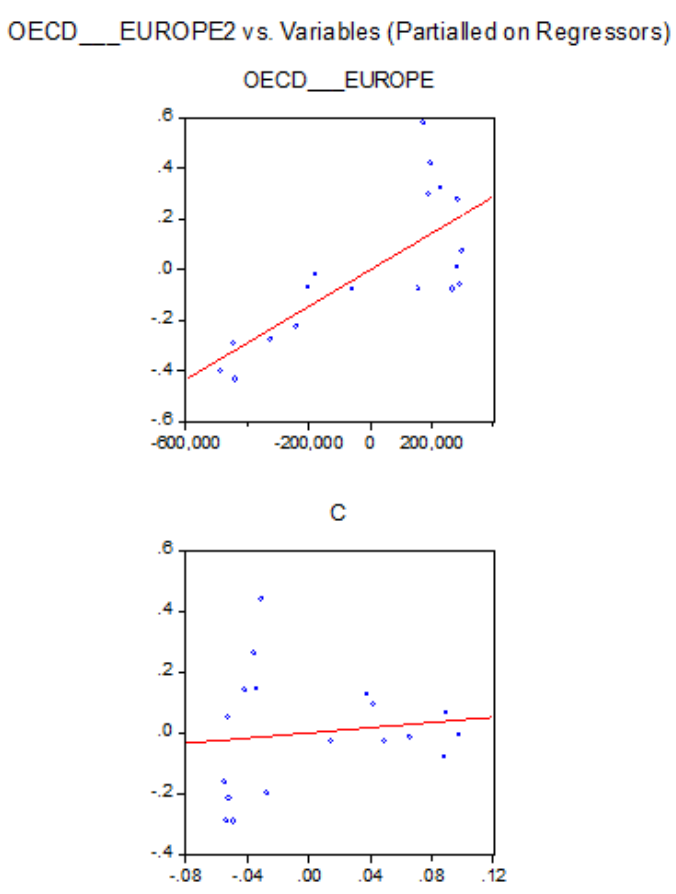

Source: Author, by using the EViews 10

The previous conclusion is confirmed by the Squared Multiple Correlation shown in following table: 


\begin{tabular}{|c|c|c|c|c|}
\hline \multicolumn{5}{|c|}{$\begin{array}{l}\text { Dependent Variable: OECD___EUROPE2 } \\
\text { Method: Least Squares } \\
\text { Date: } 04 / 11 / 19 \text { Time: } 00: 15 \\
\text { Sample: } 19992016 \\
\text { Included observations: } 18 \\
\end{array}$} \\
\hline Variable & Coefficient & Std. Error & t-Statistic & Prob. \\
\hline OECD ${ }_{C}$ EUROPE & $\begin{array}{l}7.22 \mathrm{E}-07 \\
0.429809\end{array}$ & $\begin{array}{l}1.63 \mathrm{E}-07 \\
0.837371\end{array}$ & $\begin{array}{l}4.437487 \\
0.513284\end{array}$ & $\begin{array}{l}0.0004 \\
0.6148\end{array}$ \\
\hline $\begin{array}{l}\text { R-squared } \\
\text { Adjusted R-squared } \\
\text { S.E. of regression } \\
\text { Sum squared resid } \\
\text { Log likelihood } \\
\text { F-statistic } \\
\text { Prob(F-statistic) }\end{array}$ & $\begin{array}{l}0.551711 \\
0.523693 \\
0.196495 \\
0.617767 \\
4.807244 \\
19.69129 \\
0.000414\end{array}$ & $\begin{array}{l}\text { Mean depen } \\
\text { S.D. depend } \\
\text { Akaike info c } \\
\text { Schwarz crite } \\
\text { Hannan-Quir } \\
\text { Durbin-Wats }\end{array}$ & $\begin{array}{l}\text { nt var } \\
\text { it var } \\
\text { erion } \\
\text { on } \\
\text { criter. } \\
\text { stat }\end{array}$ & $\begin{array}{r}4.139944 \\
0.284714 \\
-0.311916 \\
-0.212986 \\
-0.298275 \\
0.452208\end{array}$ \\
\hline \multicolumn{5}{|c|}{$\begin{array}{l}\text { Dependent Variable: OECD__ TOTAL2 } \\
\text { Method: Least Squares }\end{array}$} \\
\hline Variable & Coefficient & Std. Error & t-Statistic & Prob. \\
\hline OECD $]_{\mathrm{C}}$ TOTAL & $\begin{array}{r}3.05 \mathrm{E}-07 \\
-1.497109\end{array}$ & $\begin{array}{l}9.64 \mathrm{E}-08 \\
1.552867\end{array}$ & $\begin{array}{r}3.164128 \\
-0.964094\end{array}$ & $\begin{array}{l}0.0060 \\
0.3493\end{array}$ \\
\hline $\begin{array}{l}\text { R-squared } \\
\text { Adjusted R-squared } \\
\text { S.E. of regression } \\
\text { Sum squared resid } \\
\text { Log likelihood } \\
\text { F-statistic } \\
\text { Prob(F-statistic) }\end{array}$ & $\begin{array}{l}0.384892 \\
0.346448 \\
0.189145 \\
0.572416 \\
5.493462 \\
10.01170 \\
0.006014\end{array}$ & \multicolumn{2}{|c|}{$\begin{array}{l}\text { Mean dependent var } \\
\text { S.D. dependent var } \\
\text { Akaike info criterion } \\
\text { Schwarz criterion } \\
\text { Hannan-Quinn criter. } \\
\text { Durbin-Watson stat }\end{array}$} & $\begin{array}{r}3.414333 \\
0.233968 \\
-0.388162 \\
-0.289232 \\
-0.374521 \\
0.471592\end{array}$ \\
\hline
\end{tabular}

To determine the regression equation applies Least Squares Method. So, we obtain the following regression equations:

Estimation Command:

$=$ ニニニニニニニニニニニ=ニ=ニニニニニニ=ニ=

LS OECD_EUROPE2 OECD_EUROPE C

Estimation Equation:

ニニニニニニニニニニニニニニニニニニニニニニニ

OECD_EUROPE2 $=\mathrm{C}(1) * \mathrm{OECD} \_$EUROPE $+\mathrm{C}(2)$

Substituted Coefficients:

=ニニニニニニニニニニニニニニニニニ=ニニニニ=

OECD__EUROPE2 $=7.22124994626 \mathrm{e}-07 *$ OECD__EUROPE + 0.42980919739

And for the OECD Total we are obtained:

Estimation Command:

$=$ = = = = = = = = = = = = = = = = =

LS OECD__TOTAL2 OECD__TOTAL C

Estimation Equation:

ニニニニニニニニニニニニニニニニニニニニニニニ 
OECD__TOTAL2 $=\mathrm{C}(1) *$ OECD__TOTAL $+\mathrm{C}(2)$

Substituted Coefficients:

ニニニニニニニニニニニニニニニニニニニニニニニ=

OECD__TOTAL2 $=3.05152799009 \mathrm{e}-07 *$ OECD__TOTAL - 1.49710899199

The evolution of actual, fitted and residual plot for OECD Europe It can be summarized as follows:

$\begin{array}{cccc}\text { obs } & \text { Actual } & \text { Fitted } & \text { Residual } \\ 1999 & 4.72200 \ldots & 4.26620 \ldots & 0.45579 \ldots \\ 2000 & 4.561 & 4.28296 \ldots & 0.27803 \ldots \\ 2001 & 4.46400 \ldots & 4.30509 \ldots & 0.15890 \ldots \\ 2002 & 4.439 & 4.27811 \ldots & 0.16088 \ldots \\ 2003 & 4.42 & 4.34588 \ldots & 0.07411 \ldots \\ 2004 & 4.216 & 4.35550 \ldots & -0.1395 \ldots \\ 2005 & 4.151 & 4.34396 \ldots & -0.1929 \ldots \\ 2006 & 4.083 & 4.35031 \ldots & -0.2673 \ldots \\ 2007 & 4.064 & 4.33378 \ldots & -0.2697 \ldots \\ 2008 & 4.06500 \ldots & 4.25271 \ldots & -0.1877 \ldots \\ 2009 & 4.123 & 4.01126 \ldots & 0.11173 \ldots \\ 2010 & 4.064 & 4.09792 \ldots & -0.0339 \ldots \\ 2011 & 4.069 & 3.99356 \ldots & 0.07543 \ldots \\ 2012 & 3.918 & 3.96650 \ldots & -0.0485 \ldots \\ 2013 & 3.865 & 3.90616 \ldots & -0.0411 \ldots \\ 2014 & 3.74 & 3.78751 \ldots & -0.0475 \ldots \\ 2015 & 3.848 & 3.81866 \ldots & 0.02933 \ldots \\ 2016 & 3.707 & 3.82282 \ldots & -0.1158 \ldots\end{array}$

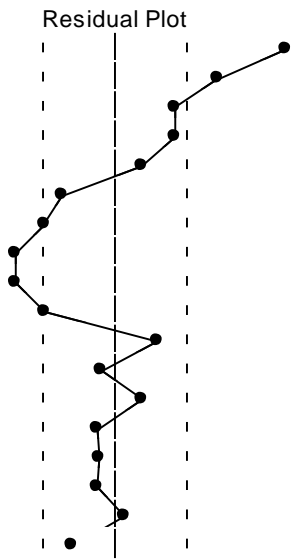

And for OECD Total, the evolution of actual, fitted and residual plot is the following:

\begin{tabular}{|c|c|c|c|c|}
\hline obs & Actual & Fitted & Residual & Residual Plot \\
\hline 1999 & 3.821 & $3.40161 \ldots$ & $0.41938 \ldots$ & 1 \\
\hline 2000 & 3.716 & $3.48620 \ldots$ & $0.22979 \ldots$ & 1 \\
\hline 2001 & 3.678 & $3.45370 \ldots$ & $0.22429 \ldots$ & 1 \\
\hline 2002 & 3.664 & $3.47684 \ldots$ & $0.18715 \ldots$ & I \\
\hline 2003 & 3.645 & $3.53091 \ldots$ & $0.11408 \ldots$ & I \\
\hline 2004 & 3.508 & $3.58571 \ldots$ & $-0.0777 \ldots$ & 1 \\
\hline 2005 & 3.476 & $3.59098 \ldots$ & $-0.1149 \ldots$ & I \\
\hline 2006 & 3.419 & $3.57345 \ldots$ & $-0.1544 \ldots$ & I \\
\hline 2007 & 3.391 & $3.63402 \ldots$ & $-0.2430 \ldots$ & । \\
\hline 2008 & 3.392 & $3.52757 \ldots$ & $-0.1355 \ldots$ & I \\
\hline 2009 & 3.402 & $3.24833 \ldots$ & $0.15366 \ldots$ & 1 \\
\hline 2010 & 3.344 & $3.39130 \ldots$ & $-0.0473 \ldots$ & ' \\
\hline 2011 & 3.394 & $3.33290 \ldots$ & $0.06109 \ldots$ & 1 \\
\hline 2012 & 3.241 & $3.26392 \ldots$ & $-0.0229 \ldots$ & I \\
\hline 2013 & 3.195 & $3.30403 \ldots$ & $-0.1090 \ldots$ & 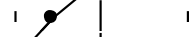 \\
\hline 2014 & 3.076 & $3.25290 \ldots$ & $-0.1769 \ldots$ & 1 \\
\hline 2015 & 3.115 & $3.22275 \ldots$ & $-0.1077 \ldots$ & 1 \\
\hline 2016 & 2.981 & $3.18077 \ldots$ & $-0.1997 \ldots$ & a \\
\hline
\end{tabular}

In the graphics bellows are actual and estimated values of the feature analysis and the residual variable values and chart series.
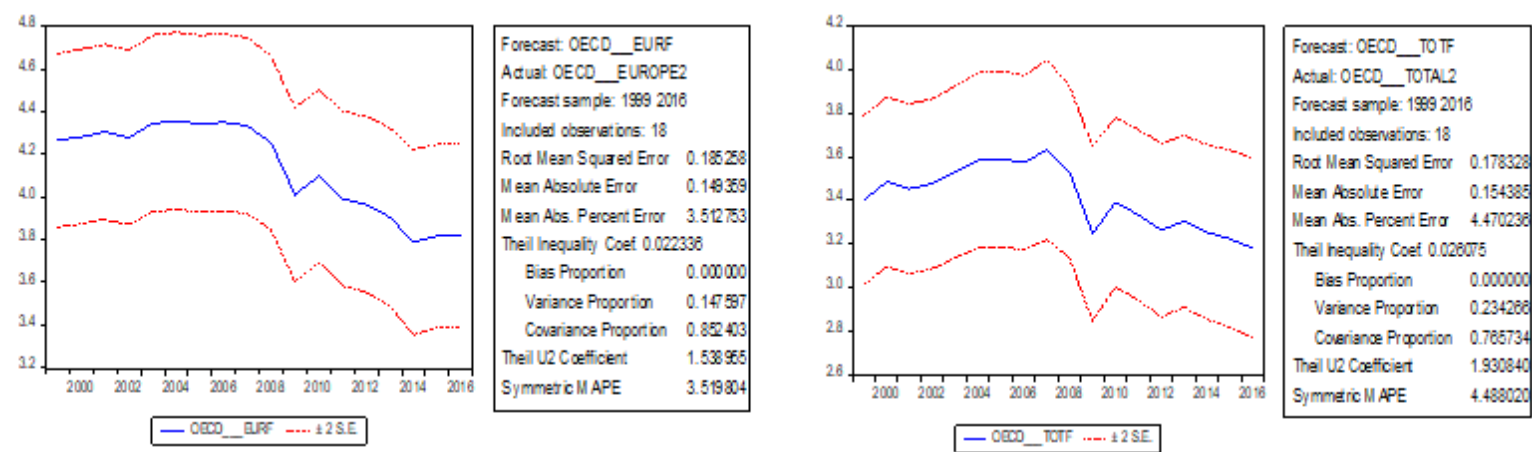

Based on the statistical data provided by OECD we analyzed with EViews and obtained the following results for OECD Europe: 

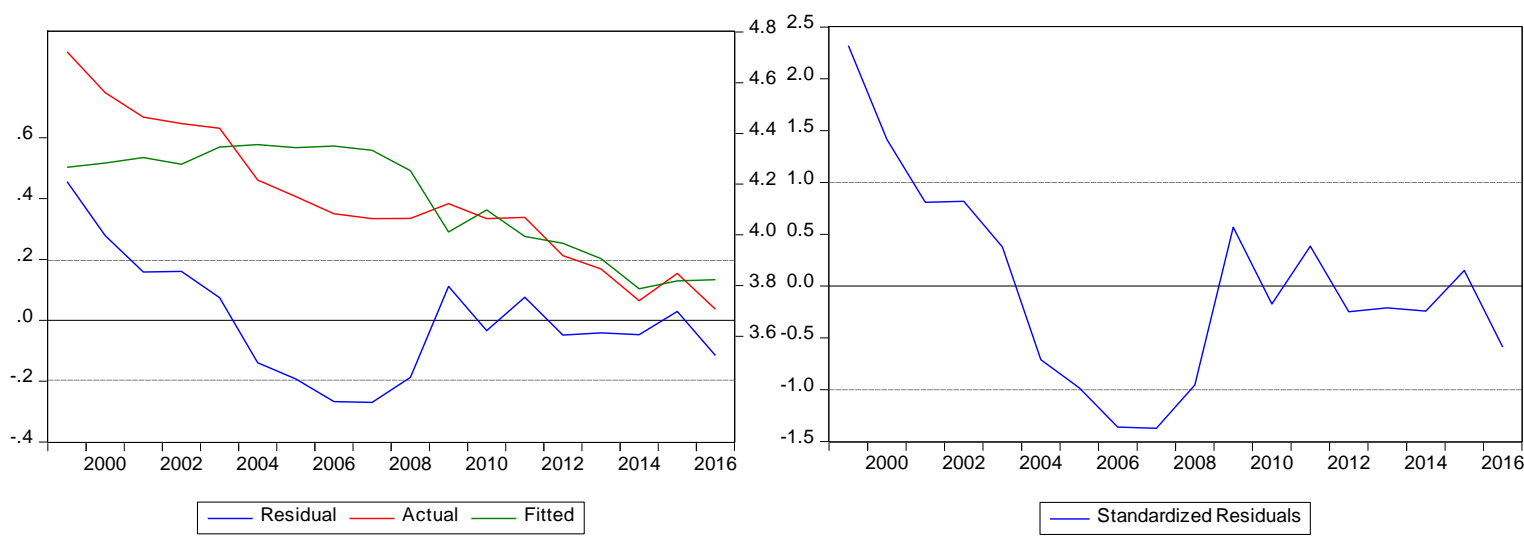

Estimation Command:

=ニニニニニニニニニニニニニニニニニニニニニニニ

LS OECD__EUROPE2 OECD__EUROPE C

Estimation Equation:

ニニニニニニニニニニニニニニニニニニニ=ニニ=ニ

OECD__EUROPE2 $=\mathrm{C}(1) * \mathrm{OECD} \_$EUROPE $+\mathrm{C}(2)$

Substituted Coefficients:

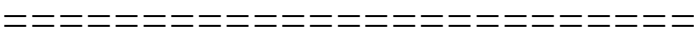

OECD_EUROPE2 $=7.22124994626 \mathrm{e}-07 *$ OECD__EUROPE + 0.42980919739

Gradients of the Objective Function

OECD_EUROPE

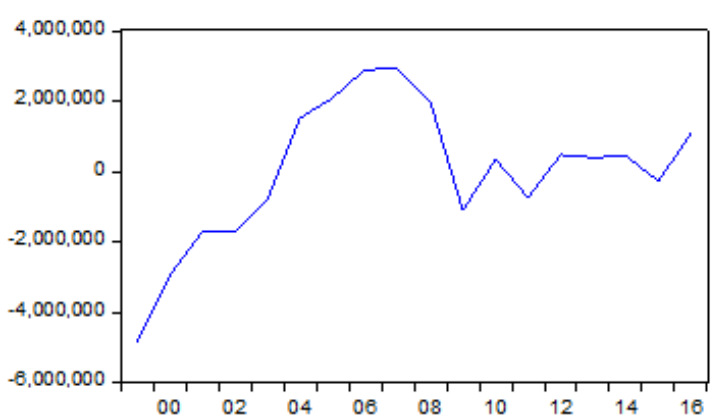

$\mathrm{c}$

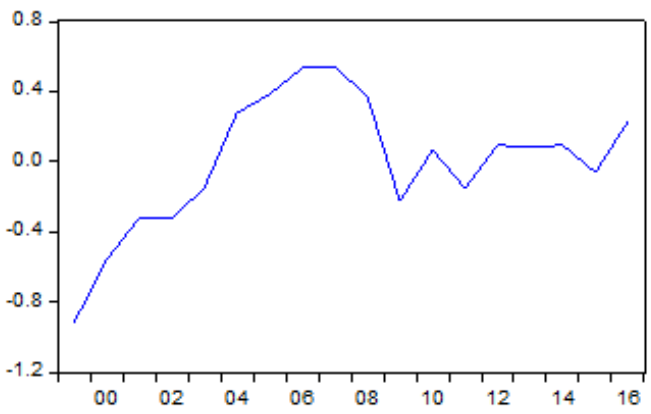

Derivatives of the Equation Specification

C(1)

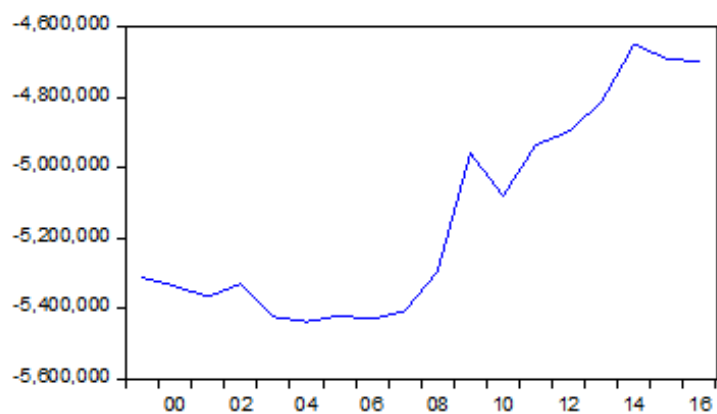

$\mathrm{C}(2)$

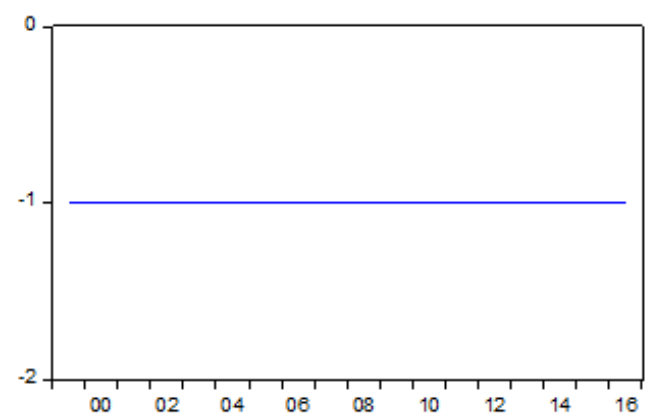


International Conference "Risk in Contemporary Economy" ISSN-L 2067-0532 ISSN online 2344-5386

$X^{\text {th }}$ Edition, 2019, Galati, Romania,

"Dunarea de Jos" University of Galati, Romania - Faculty of Economics and Business Administration

Derivatives of the Equation Specification

Equation: UNTITLED

Method: Least Squares

Specification: RESID = OECD__EUROPE2 $-\left(\mathrm{C}(1)^{\star} \mathrm{OECD} \_\right.$EUROPE $\left.+\mathrm{C}(2)\right)$

\begin{tabular}{cl}
\hline \hline Variable & Derivative of Specification \\
\hline \hline $\mathrm{C}(1)$ & - oecd__europe \\
$\mathrm{C}(2)$ & -1 \\
\hline \hline
\end{tabular}

Coefficient covariance Matrix is presented in the below table:

Derivatives of the Equation Specification

Equation: UNTITLED

Method: Least Squares

Specification: RESID = OECD__EUROPE2 $-\left(\mathrm{C}(1)^{\star} \mathrm{OECD} \_\right.$EUROPE $\left.+\mathrm{C}(2)\right)$

\begin{tabular}{cl}
\hline \hline Variable & Derivative of Specification \\
\hline \hline $\mathrm{C}(1)$ & - oecd__europe \\
$\mathrm{C}(2)$ & -1 \\
\hline \hline
\end{tabular}

Scaled Coefficients

Date: 04/11/19 Time:00:21

Sample: 19992016

Included observations: 18

\begin{tabular}{cccc}
\hline \hline Variable & Coefficient & $\begin{array}{c}\text { Standardized } \\
\text { Coefficient }\end{array}$ & $\begin{array}{c}\text { Elasticity } \\
\text { at Means }\end{array}$ \\
\hline \hline OECD__EUROPE & $7.22 \mathrm{E}-07$ & 0.742773 & 0.896180 \\
C C & 0.429809 & $\mathrm{NA}$ & 0.103820 \\
\hline \hline
\end{tabular}

Coefficient Confidence Intervals

Date: 04/11/19 Time:00:21

Sample: 19992016

Included observations: 18

\begin{tabular}{|c|c|c|c|c|c|c|c|c|}
\hline \multirow{2}{*}{\multicolumn{2}{|c|}{ Variable }} & \multirow[b]{2}{*}{ Coefficient } & \multicolumn{2}{|c|}{$90 \% \mathrm{Cl}$} & \multicolumn{2}{|c|}{$95 \% \mathrm{Cl}$} & \multicolumn{2}{|c|}{$99 \% \mathrm{Cl}$} \\
\hline & & & Low & High & Low & High & Low & High \\
\hline \multirow[t]{2}{*}{ OECD } & EUROPE & 7.22E-07 & 4.38E-07 & $1.01 \mathrm{E}-06$ & 3.77E-07 & 1.07E-06 & 2.47E-07 & 1.20E-06 \\
\hline & C & 0.429809 & -1.032143 & 1.891762 & -1.345338 & 2.204957 & -2.015969 & 2.875587 \\
\hline
\end{tabular}

Variance Inflation Factors

Date:04/11/19 Time:00:22

Sample: 19992016

Included observations: 18

\begin{tabular}{cccc}
\hline \hline Variable & $\begin{array}{c}\text { Coefficient } \\
\text { Variance }\end{array}$ & $\begin{array}{c}\text { Uncentered } \\
\text { VIF }\end{array}$ & $\begin{array}{c}\text { Centered } \\
\text { VIF }\end{array}$ \\
\hline \hline \multirow{2}{*}{ OECD_EUROPE } & $2.65 \mathrm{E}-14$ & 326.8914 & 1.000000 \\
C & 0.701190 & 326.8914 & NA \\
\hline \hline
\end{tabular}




\section{Coefficient Variance Decomposition}

Date: 04/11/19 Time: 00:23

Sample: 19992016

Included observations: 18

\begin{tabular}{|c|c|c|}
\hline $\begin{array}{c}\text { Eigenvalues } \\
\text { Condition }\end{array}$ & $\begin{array}{c}0.701190 \\
1.16 \mathrm{E}-16 \\
\end{array}$ & $\begin{array}{l}8.10 \mathrm{E}-17 \\
1.000000 \\
\end{array}$ \\
\hline \multicolumn{3}{|c|}{ Variance Decomposition Proportions } \\
\hline \multirow[b]{2}{*}{ Variable } & \multicolumn{2}{|c|}{ Associated Eigenvalue } \\
\hline & 1 & 2 \\
\hline EUROPE & 0.996941 & 0.003059 \\
\hline $\bar{C}$ & 1.000000 & $4.35 \mathrm{E}-30$ \\
\hline
\end{tabular}

Eigenvectors

\begin{tabular}{crc}
\hline \hline & \multicolumn{2}{c}{ Associated Eigenvalue } \\
Variable & 1 & 2 \\
\hline \hline OECD_EUROPE & $-1.94 \mathrm{E}-07$ & -1.000000 \\
$\mathrm{C}$ & 1.000000 & $-1.94 \mathrm{E}-07$ \\
\hline \hline
\end{tabular}

Wald Test:

Equation: Untitled

\begin{tabular}{llcc}
\hline \hline Test Statistic & Value & df & Probability \\
\hline \hline F-statistic & $3.02 \mathrm{E}+15$ & $(2,16)$ & 0.0000 \\
Chi-square & $6.05 \mathrm{E}+15$ & 2 & 0.0000 \\
\hline \hline
\end{tabular}

Null Hypothesis: $\mathrm{C}(1)=-0.7, \mathrm{C}(2)=0.4298$

Null Hypothesis Summary:

\begin{tabular}{lll}
\hline \hline Normalized Restriction $(=0)$ & Value & Std. Err. \\
\hline \hline $0.69999999999999992+C(1)$ & 0.700001 & $1.63 \mathrm{E}-07$ \\
$-0.4298+\mathrm{C}(2)$ & $9.20 \mathrm{E}-06$ & 0.837371 \\
\hline \hline
\end{tabular}

Restrictions are linear in coefficients.

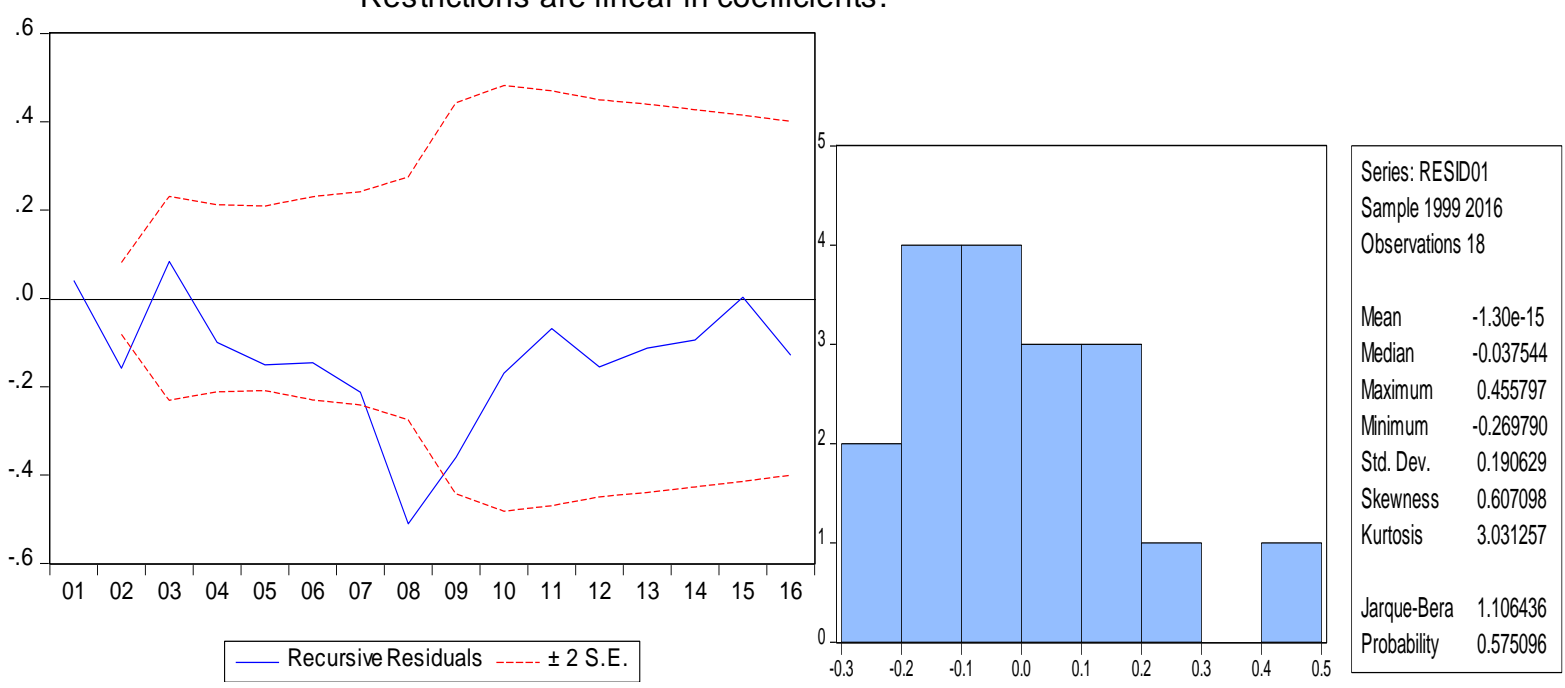


International Conference "Risk in Contemporary Economy" ISSN-L 2067-0532 ISSN online 2344-5386

$\mathrm{XX}^{\text {th }}$ Edition, 2019, Galati, Romania,

"Dunarea de Jos" University of Galati, Romania - Faculty of Economics and Business Administration

$\begin{array}{lr} & \text { RESID01 } \\ \text { Mean } & -1.30 \mathrm{E}-15 \\ \text { Median } & -0.037544 \\ \text { Maximum } & 0.455797 \\ \text { Minimum } & -0.269790 \\ \text { Std. Dev. } & 0.190629 \\ \text { Skewness } & 0.607098 \\ \text { Kurtosis } & 3.031257 \\ & \\ \text { Jarque-Bera } & 1.106436 \\ \text { Probability } & 0.575096 \\ & \\ \text { Sum } & -2.35 \mathrm{E}-14 \\ \text { Sum Sq. Dev. } & 0.617767 \\ & \\ \text { Observations } & 18\end{array}$

\section{Influence Statistics}

RStudent

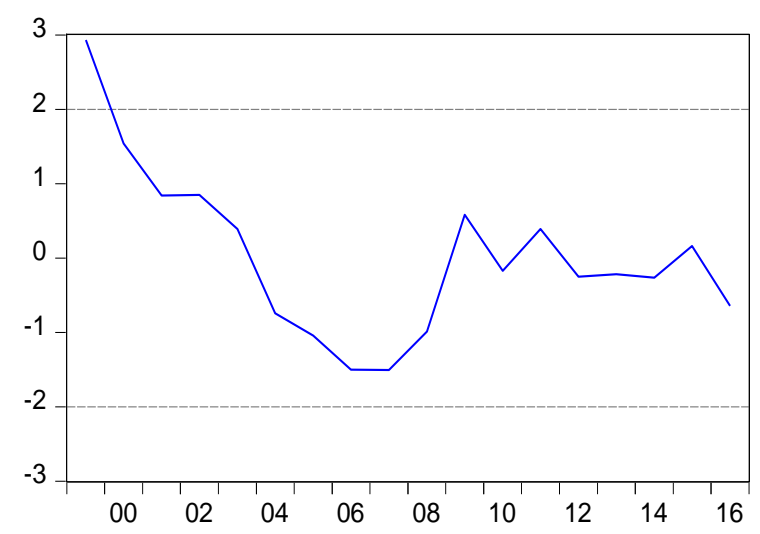

DFFITS

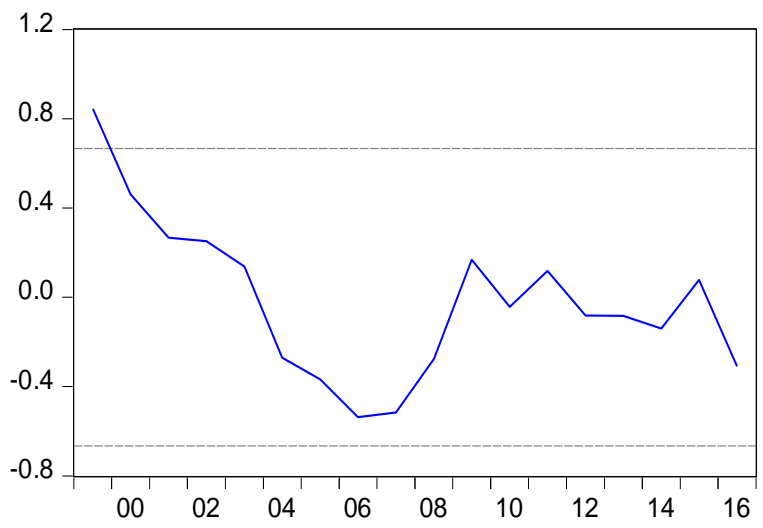

COVRATIO

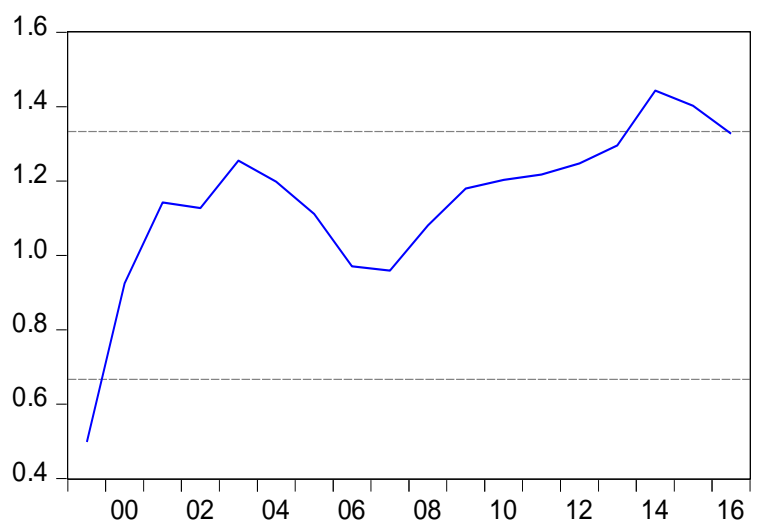


Hypothesis Testing for RESID01

Date: 04/11/19 Time:00:33

Sample: 19992016

Included observations: 18

Test of Hypothesis: Mean $=-1.30 \mathrm{e}-15$

Assuming Std. Dev. $=0.190629$

Sample Mean $=-1.30 \mathrm{e}-15$

Sample Std. Dev. $=0.190629$

\begin{tabular}{lrr} 
Method & Value & Probability \\
Z-statistic & $7.12 \mathrm{E}-17$ & 1.0000 \\
t-statistic & $7.12 \mathrm{E}-17$ & 1.0000 \\
\hline \hline Test of Hypothesis: Variance $=0.190629$ & & \\
\hline
\end{tabular}

Sample Variance $=0.036339$

\begin{tabular}{lrr}
$\frac{\text { Method }}{\text { Variance Ratio }}$ & Value & Probability \\
\hline \hline Test of Hypothesis: Median $=-0.037544$ & & \\
\hline \hline
\end{tabular}

Sample Median $=-0.037544$

Method

Sign (exact binomial)

Sign (normal approximation)

Wilcoxon signed rank

van der Waerden (normal scores)

Median Test Summary

\begin{tabular}{lrc}
\hline \hline Category & Count & Mean Rank \\
\hline \hline Obs $>-0.037544$ & 9 & 10.5555556 \\
Obs $<-0.037544$ & 9 & 8.44444444 \\
Obs $=-0.037544$ & 0 & \\
\hline \hline Total & \multicolumn{3}{c}{} \\
\hline \hline
\end{tabular}

Empirical Distribution Test for RESID01

Hypothesis: Normal

Date: 04/11/19 Time: 00:34

Sample: 19992016

Included observations: 18

\begin{tabular}{lrrr}
\hline \hline Method & Value & Adj. Value & Probability \\
\hline \hline Lilliefors (D) & 0.126170 & $\mathrm{NA}$ & $>0.1$ \\
Cramer-von Mises (W2) & 0.032044 & 0.032934 & 0.8047 \\
Watson (U2) & 0.028021 & 0.028800 & 0.8397 \\
Anderson-Darling (A2) & 0.240673 & 0.252372 & 0.7369 \\
\hline \hline
\end{tabular}

Method: Maximum Likelihood - d.f. corrected (Exact Solution)

\begin{tabular}{lrlrr}
\hline \hline Parameter & Value & Std. Error & z-Statistic & Prob. \\
\hline \hline MU & $-1.31 \mathrm{E}-15$ & 0.044932 & $-2.91 \mathrm{E}-14$ & 1.0000 \\
SIGMA & 0.190629 & 0.032693 & 5.830952 & 0.0000 \\
\hline \hline Log likelihood & 4.792818 & Mean dependent var. & $-1.30 \mathrm{E}-15$ \\
No. of Coefficients & 2 & S.D. dependent var. & 0.190629 \\
\hline \hline
\end{tabular}


Tabulation of RESID01

Date: 04/11/19 Time: 00:35

Sample: 19992016

Included observations: 18

Number of categories: 5

\begin{tabular}{c|rrrr}
\hline \hline & \multicolumn{4}{c}{ Cumulative } \\
Value & Count & Percent & Count & Percent \\
\hline$[-0.4,-0.2)$ & 2 & 11.11 & 2 & 11.11 \\
{$[-0.2,0)$} & 8 & 44.44 & 10 & 55.56 \\
{$[0,0.2)$} & 6 & 33.33 & 16 & 88.89 \\
{$[0.2,0.4)$} & 1 & 5.56 & 17 & 94.44 \\
$(0.4,0.6)$ & 1 & 5.56 & 18 & 100.00 \\
\hline Total & 18 & 100.00 & 18 & 100.00 \\
\hline \hline
\end{tabular}

BDS Test for RESID01

Date: 04/11/19 Time: 00:35

Sample: 19992016

Included observations: 18

\begin{tabular}{|c|c|c|c|c|c|}
\hline Dimension & BDS Statistic & $\underline{\text { Std. Error }}$ & z-Statistic & Prob. & \\
\hline 2 & 0.145329 & 0.018613 & 7.808120 & $\overline{0.0000}$ & \\
\hline 3 & 0.231343 & 0.030947 & 7.475379 & 0.0000 & \\
\hline 4 & 0.281990 & 0.038625 & 7.300625 & 0.0000 & \\
\hline 5 & 0.278068 & 0.042286 & 6.575836 & 0.0000 & \\
\hline 6 & 0.228340 & 0.042941 & 5.317510 & 0.0000 & \\
\hline \multicolumn{2}{|l|}{ Raw epsilon } & 0.284651 & & & \\
\hline \multicolumn{2}{|c|}{ Pairs within epsilon } & 232.0000 & V-Statistic & 0.716049 & \\
\hline \multicolumn{2}{|c|}{ Triples within epsilon } & 3214.000 & V-Statistic & 0.551097 & \\
\hline Dimension & $\underline{\mathrm{C}(\mathrm{m}, \mathrm{n})}$ & $\underline{\mathrm{c}(\mathrm{m}, \mathrm{n})}$ & $\underline{\mathrm{C}(1, n-(m-1))}$ & $\underline{c(1, n-(m-1))}$ & $\underline{\mathrm{c}(1, \mathrm{n}-(\mathrm{m}-1))^{\wedge} \mathrm{k}}$ \\
\hline 2 & 82.00000 & 0.602941 & 92.00000 & 0.676471 & 0.457612 \\
\hline 3 & 62.00000 & 0.516667 & 79.00000 & 0.658333 & 0.285323 \\
\hline 4 & 46.00000 & 0.438095 & 66.00000 & 0.628571 & 0.156106 \\
\hline 5 & 32.00000 & 0.351648 & 54.00000 & 0.593407 & 0.073580 \\
\hline 6 & 20.00000 & 0.256410 & 43.00000 & 0.551282 & 0.028070 \\
\hline
\end{tabular}

Date: 04/11/19 Time: 00:40

Sample: 19992016

Included observations: 18

Method: Holt-Winters No Seasonal

Original Series:

Forecast Series: SMOOTHED

\begin{tabular}{lrr}
\hline \hline Parameters: & Alpha & \\
Beta & 0.9400 \\
Sum of Squared Residuals & 0.1100 \\
Root Mean Squared Error & 0.278293 \\
\hline \hline & 0.124341 \\
\hline \multirow{2}{*}{ End of Period Levels: $\quad$ Mean } & -0.108282 \\
& Trend & -0.026317 \\
\hline
\end{tabular}

Based on the statistical data provided by OECD we analyzed with EViews 10 and obtained the following results for OECD Total: 

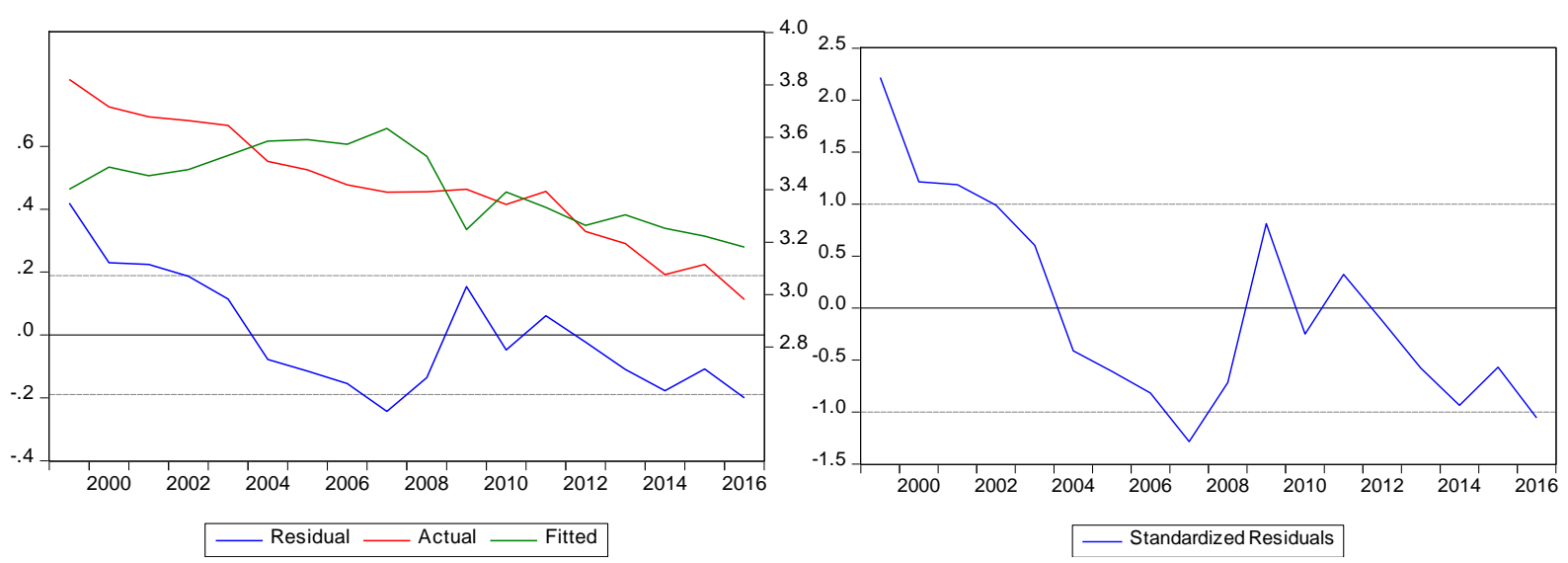

Grādients of the Objective Function

Gradients evaluated at estimated parameters

Equation: UNTITLED

Method: Least Squares

Specification: OECD__TOTAL2 OECD__TOTAL C

\begin{tabular}{|c|c|c|c|}
\hline Variable & Sum & Mean & Weighted Grad. \\
\hline OECD__TOTAL & $8.01 \mathrm{E}-08$ & 4.45E-09 & $-5.17 E-24$ \\
\hline C & $3.20 \mathrm{E}-14$ & $1.78 \mathrm{E}-15$ & $8.33 \mathrm{E}-17$ \\
\hline
\end{tabular}

Derivatives of the Equation Specification

Equation: UNTITLED

Method: Least Squares

Specification: RESID $=$ OECD

TOTAL2 - $\left(\mathrm{C}(1)^{*} \mathrm{OECD}\right.$ TOTAL $\left.+\mathrm{C}(2)\right)$

\begin{tabular}{|c|c|c|c|c|}
\hline Variable & \multicolumn{4}{|c|}{ Derivative of Specification } \\
\hline $\begin{array}{l}C(1) \\
C(2)\end{array}$ & \multicolumn{4}{|l|}{$\begin{array}{l}-0 e c d \_ \text {total } \\
-1\end{array}$} \\
\hline $\mathrm{C}^{\text {TOTAL }}$ & \multicolumn{4}{|c|}{ 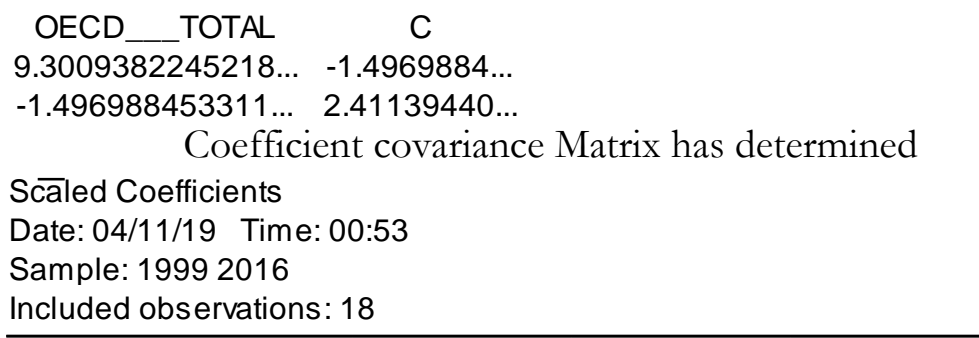 } \\
\hline & Variable & Coefficient & $\begin{array}{c}\text { Standardized } \\
\text { Coefficient }\end{array}$ & $\begin{array}{l}\text { Elasticity } \\
\text { at Means }\end{array}$ \\
\hline & OECD $]_{\mathrm{C}}$ TOTAL & $\begin{array}{r}3.05 \mathrm{E}-07 \\
-1.497109\end{array}$ & $\begin{array}{l}0.620397 \\
\text { NA }\end{array}$ & $\begin{array}{r}1.438478 \\
-0.438478\end{array}$ \\
\hline
\end{tabular}

Coefficient Confidence Intervals

Date: 04/11/19 Time: 00:53

Sample: 19992016

Included observations: 18

\begin{tabular}{crrrrrrr}
\hline & & \multicolumn{2}{c}{$90 \% \mathrm{Cl}$} & \multicolumn{2}{c}{$95 \% \mathrm{Cl}$} & \multicolumn{2}{c}{$99 \% \mathrm{Cl}$} \\
Variable & Coefficient & \multicolumn{1}{c}{ Low } & \multicolumn{1}{c}{ High } & Low & High & Low & High \\
\hline \hline \multirow{2}{*}{ OECD_TOTAL } & $3.05 \mathrm{E}-07$ & $1.37 \mathrm{E}-07$ & $4.74 \mathrm{E}-07$ & $1.01 \mathrm{E}-07$ & $5.10 \mathrm{E}-07$ & $2.35 \mathrm{E}-08$ & $5.87 \mathrm{E}-07$ \\
$\mathrm{C}$ & -1.497109 & -4.208233 & 1.214015 & -4.789039 & 1.794821 & -6.032693 & 3.038475 \\
\hline \hline
\end{tabular}


Variance Inflation Factors

Date: 04/11/19 Time: 00:54

Sample: 19992016

Included observations: 18

\begin{tabular}{cccc}
\hline \hline Variable & $\begin{array}{c}\text { Coefficient } \\
\text { Variance }\end{array}$ & $\begin{array}{c}\text { Uncentered } \\
\text { VIF }\end{array}$ & $\begin{array}{c}\text { Centered } \\
\text { VIF }\end{array}$ \\
\hline \hline OECD__TOTAL & $9.30 \mathrm{E}-15$ & 1213.247 & 1.000000 \\
$\mathrm{C}$ & 2.411394 & 1213.247 & $\mathrm{NA}$ \\
\hline \hline
\end{tabular}

Coefficient Variance Decomposition

Date: 04/11/19 Time: 00:54

Sample: 19992016

Included observations: 18

\begin{tabular}{ccc}
\hline \hline Eigenvalues & 2.411394 & $7.67 \mathrm{E}-18$ \\
Condition & $3.18 \mathrm{E}-18$ & 1.000000 \\
\hline \hline
\end{tabular}

Variance Decomposition Proportions

\begin{tabular}{ccc} 
& \multicolumn{2}{c}{ Associated Eigenvalue } \\
Variable & 1 & 2 \\
\hline \hline OECD__TOTAL & 0.999176 & 0.000824 \\
C & 1.000000 & $1.23 E-32$ \\
\hline \hline
\end{tabular}

Eigenvectors

\begin{tabular}{crc}
\hline \hline & \multicolumn{2}{c}{ Associated Eigenvalue } \\
Variable & 1 & 2 \\
\hline \hline \multirow{2}{*}{ OECD__TOTAL $_{\text {C }}$} & $-6.21 \mathrm{E}-08$ & -1.000000 \\
1.000000 & $-6.21 \mathrm{E}-08$ \\
\hline \hline
\end{tabular}

Wald Test:

Equation: Untitled

\begin{tabular}{llcc}
\hline \hline Test Statistic & Value & df & Probability \\
\hline \hline F-statistic & $3.20 \mathrm{E}+16$ & $(2,16)$ & 0.0000 \\
Chi-square & $6.39 \mathrm{E}+16$ & 2 & 0.0000 \\
\hline \hline
\end{tabular}

Null Hypothesis: $C(1)=-0.7, C(2)=-1.4971$

Null Hypothesis Summary:

\begin{tabular}{lcr}
\hline \hline Normalized Restriction $(=0)$ & Value & Std. Err. \\
\hline \hline $0.69999999999999992+\mathrm{C}(1)$ & 0.700000 & $9.64 \mathrm{E}-08$ \\
$1.4971+\mathrm{C}(2)$ & $-8.99 \mathrm{E}-06$ & 1.552867 \\
\hline \hline
\end{tabular}

Restrictions are linear in coefficients. 


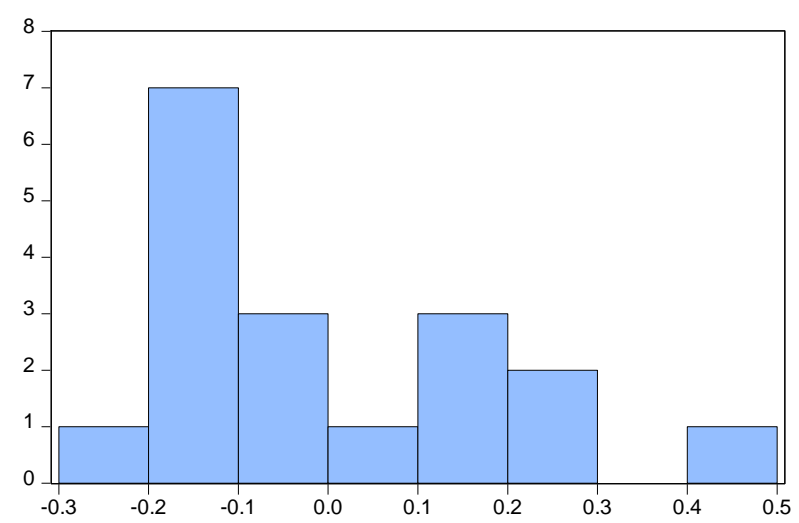

Series: Residuals

Sample 19992016

Observations 18

Mean $\quad-8.88 \mathrm{e}-16$

Median $\quad-0.062511$

Maximum 0.419388

Minimum $\quad-0.243027$

Std. Dev. $\quad 0.183498$

Skewness $\quad 0.689514$

Kurtosis $\quad 2.509604$

Jarque-Bera 1.606656

\begin{tabular}{ll} 
Probability $\quad 0.447836$ \\
\hline
\end{tabular}

Breusch-Godfrey Serial Correlation LM Test:

Null hypothesis: No serial correlation at up to 2 lags

\begin{tabular}{llll}
\hline \hline & & & \\
F-statistic & 4.431753 & Prob. F(2,14) & 0.0323 \\
Obs*R-squared & 6.978068 & Prob. Chi-Square(2) & 0.0305 \\
\hline \hline
\end{tabular}

Test Equation:

Dependent Variable: RESID

Method: Least Squares

Date:04/11/19 Time:00:59

Sample: 19992016

Included observations: 18

Presample missing value lagged residuals set to zero.

\begin{tabular}{lrlrr}
\hline \hline \multicolumn{1}{c}{ Variable } & Coefficient & Std. Error & t-Statistic & Prob. \\
\hline \hline OECD__TOTAL & $-6.53 E-08$ & $8.62 E-08$ & -0.757431 & 0.4614 \\
C RESID(-1) & 1.042035 & 1.386632 & 0.751486 & 0.4648 \\
RESID(-2) & 0.572565 & 0.267346 & 2.141664 & 0.0503 \\
\hline \hline & 0.151072 & 0.282874 & 0.534062 & 0.6017 \\
R-squared & 0.387670 & Mean dependent var & $-8.88 \mathrm{E}-16$ \\
Adjusted R-squared & 0.256457 & S.D. dependent var & 0.183498 \\
S.E. of regression & 0.158228 & Akaike info criterion & -0.656425 \\
Sum squared resid & 0.350507 & Schwarz criterion & -0.458565 \\
Log likelihood & 9.907824 & Hannan-Quinn criter. & -0.629143 \\
F-statistic & 2.954502 & Durbin-Watson stat & 1.293951 \\
Prob(F-statistic) & 0.068930 & & & \\
\hline \hline
\end{tabular}


Heteroskedasticity Test: Breusch-Pagan-Godfrey

Null hypothesis: Homoskedasticity

\begin{tabular}{llll}
\hline \hline F-statistic & 0.084874 & Prob. F $(1,16)$ & 0.7745 \\
Obs*R-squared & 0.094980 & Prob. Chi-Square(1) & 0.7579 \\
Scaled explained SS & 0.056645 & Prob. Chi-Square(1) & 0.8119 \\
\hline \hline
\end{tabular}

Test Equation:

Dependent Variable: RESID^2

Method: Least Squares

Date: 04/11/19 Time: 01:00

Sample: 19992016

Included observations: 18

\begin{tabular}{lrlrr}
\hline \hline \multicolumn{1}{c}{ Variable } & Coefficient & Std. Error & t-Statistic & Prob. \\
\hline \hline \multicolumn{1}{c}{ C } & -0.067019 & 0.339341 & -0.197498 & 0.8459 \\
\multicolumn{1}{c}{ OECD_TOTAL } & $6.14 \mathrm{E}-09$ & $2.11 \mathrm{E}-08$ & 0.291332 & 0.7745 \\
\hline \hline & 0.005277 & Mean dependent var & 0.031801 \\
R-squared & -0.056894 & S.D. dependent var & 0.040205 \\
Adjusted R-squared & 0.041333 & Akaike info criterion & -3.429868 \\
S.E. of regression & 0.027335 & Schwarz criterion & -3.330937 \\
Sum squared resid & 32.86881 & Hannan-Quinn criter. & -3.416226 \\
Log likelihood & 0.084874 & Durbin-Watson stat & 0.793522 \\
F-statistic & 0.774541 & & \\
Prob(F-statistic) & & & \\
\hline \hline
\end{tabular}

Heteroskedasticity Test: Harvey

Null hypothesis: Homoskedasticity

\begin{tabular}{llll}
\hline \hline & & & \\
F-statistic & 0.680847 & Prob. F $(1,16)$ & 0.4214 \\
Obs ${ }^{*}$ R-squared & 0.734690 & Prob. Chi-Square(1) & 0.3914 \\
Scaled explained SS & 0.258712 & Prob. Chi-Square(1) & 0.6110 \\
\hline
\end{tabular}

Test Equation:

Dependent Variable: LRESID2

Method: Least Squares

Date: 04/11/19 Time:01:00

Sample: 19992016

Included observations: 18

\begin{tabular}{lrlrr}
\hline \hline \multicolumn{1}{c}{ Variable } & Coefficient & Std. Error & t-Statistic & Prob. \\
\hline \hline \multicolumn{1}{c}{ C } & -13.37937 & 11.24233 & -1.190088 & 0.2514 \\
OECD_TOTAL & $5.76 \mathrm{E}-07$ & $6.98 \mathrm{E}-07$ & 0.825135 & 0.4214 \\
\hline \hline & & & & \\
R-squared & 0.040816 & Mean dependent var & -4.106753 \\
Adjusted R-squared & -0.019133 & S.D. dependent var & 1.356446 \\
S.E. of regression & 1.369361 & Akaike info criterion & 3.571005 \\
Sum squared resid & 30.00241 & Schwarz criterion & 3.669935 \\
Log likelihood & -30.13905 & Hannan-Quinn criter. & 3.584646 \\
F-statistic & 0.680847 & Durbin-Watson stat & 1.024941 \\
Prob(F-statistic) & 0.421428 & & \\
\hline \hline
\end{tabular}


Heteroskedasticity Test: Glejser

Null hypothesis: Homoskedasticity

\begin{tabular}{llll}
\hline \hline F-statistic & 0.267490 & Prob. F $(1,16)$ & 0.6121 \\
Obs*R-squared & 0.295978 & Prob. Chi-Square(1) & 0.5864 \\
Scaled explained SS & 0.181362 & Prob. Chi-Square(1) & 0.6702 \\
\hline \hline
\end{tabular}

Test Equation:

Dependent Variable: ARESID

Method: Least Squares

Date: 04/11/19 Time: 01:00

Sample: 19992016

Included observations: 18

\begin{tabular}{lrlrr}
\hline \multicolumn{1}{c}{ Variable } & Coefficient & Std. Error & t-Statistic & Prob. \\
\hline \hline \multicolumn{1}{c}{ C } & -0.244097 & 0.770787 & -0.316685 & 0.7556 \\
OECD_TOTAL & $2.48 E-08$ & $4.79 E-08$ & 0.517194 & 0.6121 \\
\hline \hline R-squared & 0.016443 & Mean dependent var & 0.154385 \\
Adjusted R-squared & -0.045029 & S.D. dependent var & 0.091840 \\
S.E. of regression & 0.093885 & Akaike info criterion & -1.789053 \\
Sum squared resid & 0.141030 & Schwarz criterion & -1.690123 \\
Log likelihood & 18.10148 & Hannan-Quinn criter. & -1.775412 \\
F-statistic & 0.267490 & Durbin-Watson stat & 0.781161 \\
Prob(F-statistic) & 0.612096 & & \\
\hline \hline & & & \\
Heteroskedasticity Test: ARCH & 5.627708 & Prob. F $(1,15)$ & \\
\hline \hline
\end{tabular}

Test Equation:

Dependent Variable: RESID^2

Method: Least Squares

Date: 04/11/19 Time: 01:01

Sample (adjusted): 20002016

Included observations: 17 after adjus tments

\begin{tabular}{lrlrr}
\hline \hline \multicolumn{1}{c}{ Variable } & Coefficient & Std. Error & t-Statistic & Prob. \\
\hline \hline \multicolumn{1}{c}{ C } & 0.015998 & 0.005022 & 3.185569 & 0.0061 \\
\multicolumn{1}{c}{ RESID^2(-1) } & 0.233931 & 0.098610 & 2.372279 & 0.0315 \\
\hline \hline & & & & \\
R-squared & 0.272823 & Mean dependent var & 0.023325 \\
Adjusted R-squared & 0.224344 & S.D. dependent var & 0.018537 \\
S.E. of regression & 0.016326 & Akaike info criterion & -5.281997 \\
Sum squared resid & 0.003998 & Schwarz criterion & -5.183971 \\
Log likelihood & 46.89697 & Hannan-Quinn criter. & -5.272253 \\
F-statistic & 5.627708 & Durbin-Watson stat & 1.938800 \\
Prob(F-statistic) & 0.031483 & & \\
\hline \hline
\end{tabular}


Heteroskedasticity Test: White

Null hypothesis: Homoskedasticity

\begin{tabular}{llll}
\hline \hline & & & \\
F-statistic & 0.312540 & Prob. F(2,15) & 0.7362 \\
Obs*R-squared & 0.720089 & Prob. Chi-Square(2) & 0.6976 \\
Scaled explained SS & 0.429451 & Prob. Chi-Square(2) & 0.8068 \\
\hline \hline
\end{tabular}

Test Equation:

Dependent Variable: RESID^2

Method: Least Squares

Date: 04/11/19 Time: 01:01

Sample: 19992016

Included observations: 18

\begin{tabular}{lrllr}
\hline \hline \multicolumn{1}{c}{ Variable } & Coefficient & Std. Error & t-Statistic & Prob. \\
\hline \hline \multicolumn{1}{c}{ C } & -11.17235 & 15.07967 & -0.740888 & 0.4702 \\
OECD_TOTAL^2 & $-4.30 \mathrm{E}-14$ & $5.84 \mathrm{E}-14$ & -0.736636 & 0.4727 \\
OECD_TOTAL & $1.39 \mathrm{E}-06$ & $1.88 \mathrm{E}-06$ & 0.739858 & 0.4708 \\
\hline \hline & & & & \\
R-squared & 0.040005 & Mean dependent var & 0.031801 \\
Adjusted R-squared & -0.087994 & S.D. dependent var & 0.040205 \\
S.E. of regression & 0.041937 & Akaike info criterion & -3.354293 \\
Sum squared resid & 0.026380 & Schwarz criterion & -3.205898 \\
Log likelihood & 33.18864 & Hannan-Quinn criter. & -3.333831 \\
F-statistic & 0.312540 & Durbin-Watson stat & 0.931759 \\
Prob(F-statistic) & 0.736237 & & \\
\hline \hline
\end{tabular}

Heteroskedasticity Test: Breusch-Pagan-Godfrey

Null hypothesis: Homoskedasticity

\begin{tabular}{llll}
\hline \hline F-statistic & 3.511358 & Prob. F(3,13) & 0.0462 \\
Obs ${ }^{*}$-squared & 7.609361 & Prob. Chi-Square(3) & 0.0548 \\
Scaled explained SS & 0.961373 & Prob. Chi-Square(3) & 0.8106 \\
\hline \hline
\end{tabular}

Test Equation:

Dependent Variable: RESID^2

Method: Least Squares

Date: 04/11/19 Time:01:02

Sample (adjusted): 20002016

Included observations: 17 after adjustments

\begin{tabular}{crlrr}
\hline \hline \multicolumn{1}{c}{ Variable } & Coefficient & Std. Error & t-Statistic & Prob. \\
\hline \hline C & 12.27923 & 6.196047 & 1.981784 & 0.0691 \\
RESID^2(-1) & 0.297487 & 0.101919 & 2.918850 & 0.0120 \\
OECD__TOTAL^2 & $4.76 \mathrm{E}-14$ & $2.40 \mathrm{E}-14$ & 1.987968 & 0.0683 \\
OECD__TOTAL & $-1.53 \mathrm{E}-06$ & $7.71 \mathrm{E}-07$ & -1.983675 & 0.0688 \\
\hline \hline & & & & \\
R-squared & 0.447609 & Mean dependent var & 0.023325 \\
Adjusted R-squared & 0.320135 & S.D. dependent var & 0.018537 \\
S.E. of regression & 0.015285 & Akaike info criterion & -5.321617 \\
Sum squared resid & 0.003037 & Schwarz criterion & -5.125567 \\
Log likelihood & 49.23375 & Hannan-Quinn criter. & -5.302130 \\
F-statistic & 3.511358 & Durbin-Watson stat & 2.045095 \\
Prob(F-statistic) & 0.046249 & & \\
\hline \hline
\end{tabular}


Heteroskedasticity Test: Harvey

Null hypothesis: Homoskedasticity

\begin{tabular}{llll}
\hline \hline & & & \\
F-statistic & 1.985455 & Prob. F $(3,13)$ & 0.1660 \\
Obs ${ }^{*}$ R-squared & 5.341647 & Prob. Chi-Square(3) & 0.1484 \\
Scaled explained SS & 1.613328 & Prob. Chi-Square(3) & 0.6564 \\
\hline \hline
\end{tabular}

Test Equation:

Dependent Variable: LRESID2

Method: Least Squares

Date: 04/11/19 Time: $01: 03$

Sample (adjusted): 20002016

Included observations: 17 after adjustments

\begin{tabular}{crrrr}
\hline \hline \multicolumn{1}{c}{ Variable } & Coefficient & Std. Error & t-Statistic & Prob. \\
\hline \hline C & 522.7823 & 439.6592 & 1.189062 & 0.2557 \\
LRESID2(-1) & 0.442146 & 0.223463 & 1.978607 & 0.0694 \\
OECD__TOTAL2 & $2.05 E-12$ & $1.70 \mathrm{E}-12$ & 1.203077 & 0.2504 \\
OECD_TOTAL & $-6.56 \mathrm{E}-05$ & $5.47 \mathrm{E}-05$ & -1.199040 & 0.2519 \\
\hline \hline & & & & \\
R-squared & 0.314215 & Mean dependent var & -4.246096 \\
Adjusted R-squared & 0.155956 & S.D. dependent var & 1.258413 \\
S.E. of regression & 1.156127 & Akaike info criterion & 3.330353 \\
Sum squared resid & 17.37619 & Schwarz criterion & 3.526403 \\
Log likelihood & -24.30800 & Hannan-Quinn criter. & 3.349840 \\
F-statistic & 1.985455 & Durbin-Watson stat & 2.252704 \\
Prob(F-statistic) & 0.166007 & & \\
\hline \hline
\end{tabular}

Heteroskedasticity Test: Glejser

Null hypothesis: Homoskedasticity

\begin{tabular}{llll}
\hline \hline F-statistic & 3.819468 & Prob. F(3,13) & 0.0366 \\
Obs ${ }^{R}$-squared & 7.964251 & Prob. Chi-Square(3) & 0.0468 \\
Scaled explained SS & 2.487632 & Prob. Chi-Square(3) & 0.4775 \\
\hline \hline
\end{tabular}

Test Equation:

Dependent Variable: ARESID

Method: Least Squares

Date: 04/11/19 Time: 01:03

Sample (adjusted): 20002016

Included observations: 17 after adjustments

\begin{tabular}{lrlrr}
\hline \hline \multicolumn{1}{c}{ Variable } & Coefficient & Std. Error & t-Statistic & Prob. \\
\hline \hline C & 42.66594 & 21.15907 & 2.016438 & 0.0649 \\
ARESID(-1) & 0.471562 & 0.156100 & 3.020896 & 0.0098 \\
OECD__TOTAL^2 & $1.65 \mathrm{E}-13$ & $8.18 \mathrm{E}-14$ & 2.017750 & 0.0647 \\
OECD__TOTAL & $-5.31 \mathrm{E}-06$ & $2.63 \mathrm{E}-06$ & -2.015268 & 0.0650 \\
\hline \hline & & & & \\
R-squared & 0.468485 & Mean dependent var & 0.138797 \\
Adjusted R-squared & 0.345828 & S.D. dependent var & 0.065684 \\
S.E. of regression & 0.053126 & Akaike info criterion & -2.829975 \\
Sum squared resid & 0.036691 & Schwarz criterion & -2.633925 \\
Log likelihood & 28.05479 & Hannan-Quinn criter. & -2.810487 \\
F-statistic & 3.819468 & Durbin-Watson stat & 2.214290 \\
Prob(F-statistic) & 0.036632 & & & \\
\hline \hline
\end{tabular}




\begin{tabular}{|c|c|c|c|c|}
\hline \multicolumn{5}{|c|}{$\begin{array}{l}\text { Ramsey RESET Test } \\
\text { Equation: UNTITLED } \\
\text { Omitted Variables: Squares of fitted values } \\
\text { Specification: OECD__TOTAL2 OECD__TOTAL C }\end{array}$} \\
\hline & Value & $\mathrm{df}$ & Probability & \\
\hline t-statistic & 3.936943 & 15 & 0.0013 & \\
\hline F-statistic & 15.49952 & $(1,15)$ & 0.0013 & \\
\hline Likelihood ratio & 12.77390 & 1 & 0.0004 & \\
\hline \multicolumn{5}{|l|}{ F-test summary: } \\
\hline & Sum of Sa. & $\mathrm{df}$ & Mean Square & \\
\hline Test SSR & 0.290895 & 1 & 0.290895 & \\
\hline Restricted SSR & 0.572416 & 16 & 0.035776 & \\
\hline Unrestricted SSR & 0.281520 & 15 & 0.018768 & \\
\hline \multicolumn{5}{|l|}{ LR test summary: } \\
\hline & Value & & & \\
\hline Restricted LogL & 5.493462 & & & \\
\hline Unrestricted LogL & 11.88041 & & & \\
\hline $\begin{array}{l}\text { Unrestricted Test Equ } \\
\text { Dependent Variable: } \\
\text { Method: Least Square } \\
\text { Date: } 04 / 11 / 19 \text { Time: } \\
\text { Sample: } 19992016 \\
\text { Included observations }\end{array}$ & : 04 & & & \\
\hline Variable & Coefficient & Std. Error & $\mathrm{t}$-Statistic & Prob. \\
\hline OECD__ TOTAL & $1.71 \mathrm{E}-05$ & 4.26E-06 & 4.008017 & 0.0011 \\
\hline $\mathrm{C}$ & -177.3042 & 44.66990 & -3.969210 & 0.0012 \\
\hline FITTED^2 & -8.067122 & 2.049083 & -3.936943 & 0.0013 \\
\hline R-squared & 0.697483 & \multicolumn{2}{|c|}{ Mean dependent var } & 3.414333 \\
\hline Adjusted R-squared & 0.657148 & \multicolumn{2}{|c|}{ S.D. dependent var } & 0.233968 \\
\hline S.E. of regression & 0.136996 & \multicolumn{2}{|c|}{ Akaike info criterion } & -0.986712 \\
\hline Sum squared resid & 0.281520 & \multicolumn{2}{|c|}{ Schwarz criterion } & -0.838317 \\
\hline Log likelihood & 11.88041 & \multicolumn{2}{|c|}{ Hannan-Quinn criter. } & -0.966251 \\
\hline F-statistic & 17.29202 & \multicolumn{2}{|c|}{ Durbin-Wats on stat } & 1.644629 \\
\hline Prob(F-statistic) & 0.000128 & & & \\
\hline
\end{tabular}




\section{Influence Statistics}

\section{RStudent}

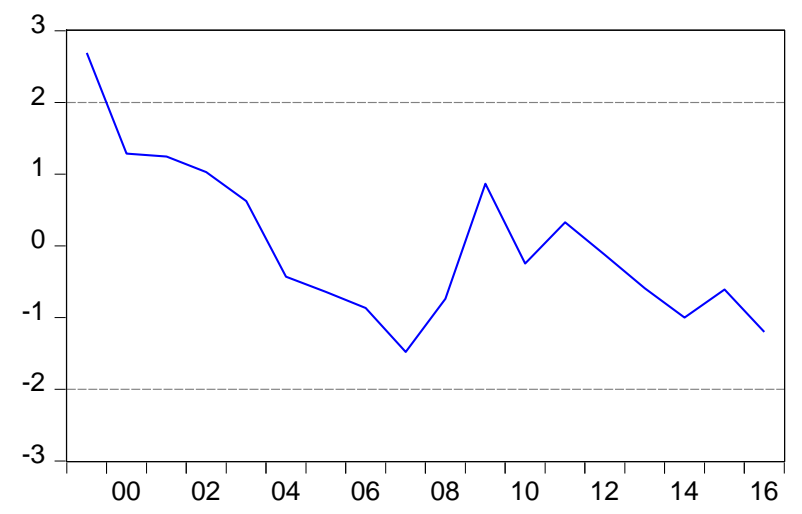

DFFITS

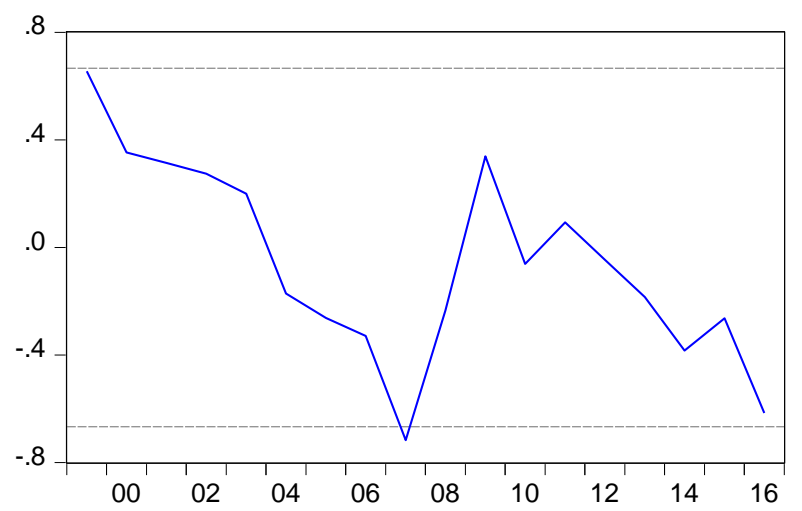

COVRATIO

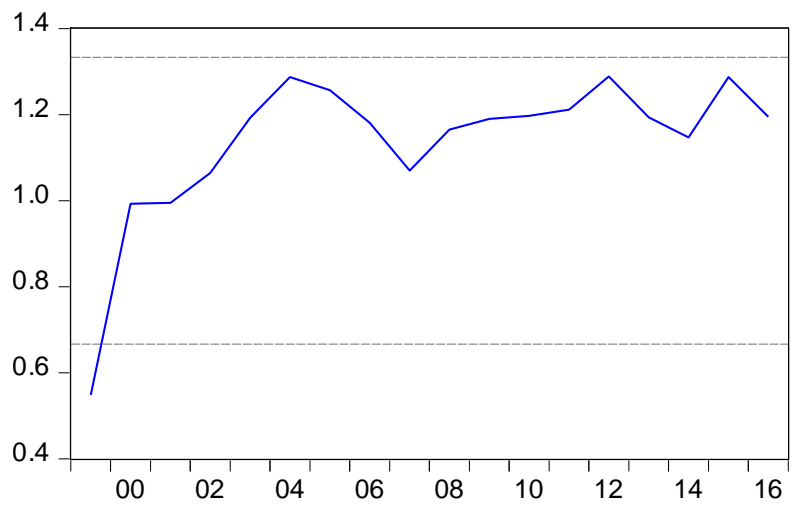

The estimation for OECD Total for mortality and welfare costs is the following: 


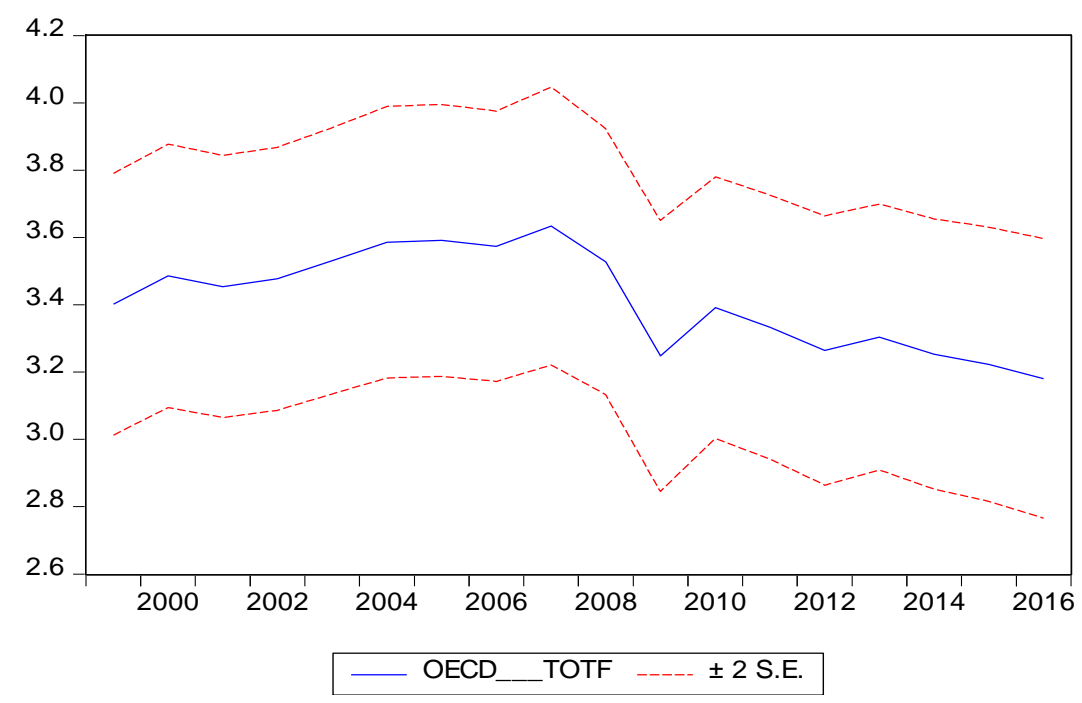

\begin{tabular}{|c|c|}
\hline \multicolumn{2}{|l|}{ Forecast: OECD_TOTF } \\
\hline \multicolumn{2}{|l|}{ Actual: OECD__TOTAL2 } \\
\hline \multicolumn{2}{|l|}{ Forecast sample: 19992016} \\
\hline \multicolumn{2}{|l|}{ Included observations: 18} \\
\hline Root Mean Squared Error & 0.178328 \\
\hline Mean Absolute Error & 0.154385 \\
\hline Mean Abs. Percent Error & 4.470236 \\
\hline \multicolumn{2}{|c|}{ Theil Inequality Coef. 0.026075} \\
\hline Bias Proportion & 0.000000 \\
\hline Variance Proportion & 0.234266 \\
\hline Covariance Proportion & 0.765734 \\
\hline Theil U2 Coefficient & 1.930840 \\
\hline Symmetric MAPE & 4.488020 \\
\hline
\end{tabular}

Date: 04/11/19 Time:01:08

Sample: 19992016

Included observations: 18

Method: Holt-Winters No Seasonal

Original Series:

Forecast Series: SMOOTHED

\begin{tabular}{llr}
\hline \hline Parameters: & Alpha & \\
& Beta & 0.8800 \\
Sum of Squared Residuals & 0.0100 \\
Root Mean Squared Error & 0.254604 \\
End of Period Levels: $\quad$ Mean & 0.118931 \\
& Trend & -0.197424 \\
& Trend \\
\hline \hline
\end{tabular}

\section{Conclusions}

Global climate is in constant change which affects both the health of the world population, and the level of public expenditure. Greenhouse gases have implications on the environment with potentially damaging effects on ecosystems, biodiversity and the means of human subsistence. Air pollution is a consequence of increasing urbanization and industrialization. Also, greenhouse gas (GHG) emissions contribute to accelerating climate change.

The global climate change has an impact on mortality rate, public health and welfare cost from exposure to environmental risks. It is a dynamic causal relationship between greenhouse gases, mortality rate and public health and welfare cost, shows by the time-series data for the period $1999-2016$.

Experts say that if greenhouse gas emissions continue at their rate in 2017, the Earth's surface temperature could exceed historic values from 2047 with potentially damaging effects on ecosystems, biodiversity and the means of human subsistence.

\section{References}

1. Bates D.V, (995), Health impacts of air pollution-continuing problems, Scand J Work. Environ Health.

2. Dragomir, S., Dragomir, G., Trandafir, M., Necsulescu, E., Pripoaie, R., Cretu, C., \& Turtureanu, A. (2010). LANDFIL FOR WASTE DEPOSING IN EFFICIENTLY CONDITIONS. International Multidisciplinary Scientific GeoConference: SGEM: Surveying Geology \& mining Ecology Management, 2, 635.

3. Wong, E, Goblke, J, Griffith, W, Farrow, S and Faustman, E, (2004), "Assessing the Health Benefits of Air Pollution Reduction for Children", Environmental Health Perspectives, VOLUME 112, NUMBER 2. 
International Conference "Risk in Contemporary Economy" ISSN-L 2067-0532 ISSN online 2344-5386

$X^{\text {th }}$ Edition, 2019, Galati, Romania,

"Dunarea de Jos" University of Galati, Romania - Faculty of Economics and Business Administration

4. https:// ec.europa.eu/eurostat/statistics-explained/index.php/_Air_pollution_statistics__air_emissions_accounts

5. bttps:// wnw.epa.gov/ghgemissions/global-greenhouse-gas-emissions-data

6. http:// wnw.europarl.europa.eu/news/en/ headlines/society/20180301STO98928/greenhouse-gasemissions-by-country-and-sector-infographic

7. http:// wnw.europarl.europa.eu/news/en/ beadlines/society/201807035TO07123/ climate-change-ineurope-facts-and-figures

8. http:// www.europarl.europa.eu/news/en/ headlines/society/20180208STO97442/cutting-eu-greenhousegas-emissions-national-targets-for-2030

9. bttps:// wmw.greenpeace.org/romania/ro/campanii/paduri/Activitati/dezvoltare-fara-defrisare/

10. www.oecd.org

11. bttps:// ourworldindata.org/air-pollution

12. bttps:// wmw.stateofglobalair.org/data/

13. bttps:// unfccc.int/process-and-meetings/transparency-and-reporting/greenhouse-gas-data/ghg-dataunfccc/ghg-data-from-unfccc

14. bttps:// unfccc.int/ topics/land-use/workstreams/land-use--land-use-change-and-forestry-lulucf

15. bttps:// di.unfccc.int/global_map_content/map/index.btml?year=growth_base_finalergas=Aggregate_GH Gsesector $=$ to\& room $=2$ \&lat $=30 \mho \mathrm{lng}=15$

16. https:// waqi.info/ 